ARTICLE

https://doi.org/10.1038/s41467-020-14985-8

\title{
Maltotriose-based probes for fluorescence and photoacoustic imaging of bacterial infections
}

\author{
Aimen Zlitni (1) 1,2, Gayatri Gowrishankar ${ }^{1,2}$, Idan Steinberg (10) 1,2, Tom Haywood ${ }^{1,2}$ \& Sanjiv Sam Gambhir (i) 1,2,3凶
}

Currently, there are no non-invasive tools to accurately diagnose wound and surgical site infections before they become systemic or cause significant anatomical damage. Fluorescence and photoacoustic imaging are cost-effective imaging modalities that can be used to noninvasively diagnose bacterial infections when paired with a molecularly targeted infection imaging agent. Here, we develop a fluorescent derivative of maltotriose (Cy7-1-maltotriose), which is shown to be taken up in a variety of gram-positive and gram-negative bacterial strains in vitro. In vivo fluorescence and photoacoustic imaging studies highlight the ability of this probe to detect infection, assess infection burden, and visualize the effectiveness of antibiotic treatment in E. coli-induced myositis and a clinically relevant S. aureus wound infection murine model. In addition, we show that maltotriose is an ideal scaffold for infection imaging agents encompassing better pharmacokinetic properties and in vivo stability than other maltodextrins (e.g. maltohexose).

\footnotetext{
${ }^{1}$ Molecular Imaging Program at Stanford, Stanford University, Stanford, CA 94305, USA. ${ }^{2}$ Department of Radiology, Stanford University, Stanford, CA 94305, USA. ${ }^{3}$ Department of Bioengineering, Department of Materials Science \& Engineering, Stanford University, Stanford, CA 94305, USA.

凶email: sgambhir@stanford.edu
} 
B acterial infections are of mounting medical and public concern worldwide. One primary reason for this epidemic is the overuse of antimicrobials, which enhanced the number of drug-resistant bacteria ${ }^{1,2}$. Furthermore, there is an increase in human life expectancy, which contributes to the high number of individuals at risk for infection and the proliferation of necessary medical procedures (i.e. surgery, arthroplasty, fracture fixations, biomedical implantations) ${ }^{3}$. Such procedures and associated implants are susceptible to infection ${ }^{4-7}$.

Wound and surgical infections create a substantial burden on patients' quality of life and result in delayed healing and can even lead to death ${ }^{7}$. For example, surgical site infections (SSIs) are one of the most common types of healthcare-associated infections and occur in $2-5 \%$ of patients undergoing surgery in the United States. This translates to around 400,000 SSIs for an average of 15 million procedures performed annually in the United States. In addition to increasing the duration of hospitalization, SSIs increase treatment cost as well as mortality risk by $2-11$-fold $^{8}$. Unfortunately, many of these infections are only diagnosed after becoming systemic or having caused significant damage to key organs, making it harder and more costly to treat due to the high bacterial burden. It is therefore important to develop tools to noninvasively detect bacterial infections at an early stage with high sensitivity and specificity. Such tools will aid clinicians in deciding the optimal route of treatment after surgeries and can be used to monitor the effectiveness of the treatment regimen to ensure proper management of wound and surgical infections. In the clinic, bacterial infections are diagnosed through a combination of clinical, laboratory (detecting signs of inflammation, microbiology, and histopathology), and imaging assessments which are invasive, time-consuming, and/or costly6,7,9. Currently, imaging modalities such as X-ray, ultrasound, magnetic resonance imaging (MRI), and computed tomography (CT) provide valuable anatomical information but are only useful in diagnosing delayed and late-stage infections ${ }^{9}$. In order to improve sensitivity, specificity, and earlier detection, a molecular imaging strategy, which necessitates the development of imaging probes that can specifically target sites of bacterial infections, will be required. A variety of radio-imaging agents or positron emission tomography (PET) tracers for whole-body bacterial imaging have been developed and several of these are currently under evaluation in clinical trials ${ }^{10}$. These approaches are however dependent on proximity to cyclotrons and generators (for isotope production or collection, respectively) and experienced radiochemists for tracer production, thus limiting their availability on demand. Their use will therefore likely be restricted to major hospitals for the management of in-patients. In addition, such approaches are not recommended for use with infants, children, and pregnant women, who represent a population at high risk for infections, due to radiation exposure. To date, there are no rapid and reliable diagnostic techniques that can detect implant, wound, and surgical infections at an early stage in outpatient clinics. More specifically, an imaging tool that can aid doctors in emergency rooms (ERs) and field hospitals to quickly diagnose bacterial wound infections and determine the extent of infection can potentially change clinical management.

Fluorescence imaging (FLI) relies on the detection of emission signals from fluorescent probes upon excitation at their appropriate absorbance wavelengths ${ }^{11,12}$. FLI of bacterial infections gained attention due to its many advantages such as high resolution, real-time imaging capabilities, ease of use, and low cost $\mathrm{t}^{11}$. Constricted by its limited depth and penetration $(\sim 1 \mathrm{~cm})$, we believe that FLI can only be implemented in superficial infection imaging (during surgery, superficial implants, or endoscopy) as well as intra-operative applications ${ }^{11,12}$. On the other hand, photoacoustic imaging (PAI) is an emerging imaging technique that relies on detecting ultrasound signals produced upon thermal expansion of tissue when exciting the fluorescent probe at an appropriate wavelength with an external laser ${ }^{13}$. PAI has been shown to be a standalone, portable tool capable of imaging endogenous signals such as melanin, and exogenous chromophores from contrast agents, with deeper imaging capabilities (up to $4 \mathrm{~cm}$ ) than FLI and comparable resolution to MRI $(\sim 250 \mu \mathrm{m})^{13-16}$. In addition, PAI has been used to monitor tissue healing by imaging blood vessels as well as utilizing its ultrasound component to provide anatomical information ${ }^{15-18}$. Hence, PAI can be an optimal cost-effective and non-invasive tool to quickly detect bacterial infections and monitor the effectiveness of treatment at local sites (i.e. surgery and injury sites).

A number of FLI and/or PAI probes targeted to bacteria have been developed by using antibiotics (vancomycin ${ }^{19,20}$ or teicoplanin $^{21}$, specific to Gram-positive bacteria), Concanavalin (targeting bacterial cell-surface mannose) ${ }^{22}$, antibodies (targeting the immunodominant staphylococcal antigen A, specific to $S$. aureus $)^{23}$, boronic-acid (targeting bacterial cell-surface glycoproteins, specific to Gram-positive bacteria) ${ }^{24}$, enzyme-activated nanoparticles (targeting gelatinase-expressing Gram-positive bacteria $)^{20}$ or through electrostatic and hydrophobic interactions (specific to Gram-positive bacteria) ${ }^{25}$. Preclinical evaluation of these probes showed promising results in FLI ${ }^{19,22-24}$ or $\mathrm{PAI}^{20,21}$ of some bacterial infections. Unfortunately, targeting the bacterial cell wall potentially limits the amount of signaling agent taken up leading to lower sensitivity. In addition, strain-specific probes will have minimal clinical impact in imaging surgery and injury-related bacterial infections since they usually occur from the presence of a variety of pathogenic bacteria. A few other examples rely on genetically encoding bacteria with reporters, such as photo-switchable chromoproteins ${ }^{26,27}$ and violacein ${ }^{28}$, have been reported. While these strategies allow non-invasive imaging of bacteria in vivo using PAI, the application of such platform would be limited to visualizing biochemistry, pathophysiological processes, and gene expression profiles in living subjects as well as imaging tumor homing bacteria ${ }^{29}$ and cannot be used for diagnosing bacterial infections.

A more promising bacterial-imaging strategy relies on the utility of large sugar molecules to deliver the signaling moiety into bacteria. These complex sugars (e.g. maltose, maltotriose, and maltohexose) are major sources of glucose for bacteria and are taken up in millimolar quantities ${ }^{30}$. A considerable advantage of such probes is their specific uptake by bacteria through the maltodextrin transporter which is not present in mammalian cells, thus allowing differentiation of bacterial infections from other diseases such as cancer and inflammation. Murthy and coworkers ${ }^{30}$ developed a fluorescent and an ${ }^{18} \mathrm{~F}$-labeled ${ }^{31}$ derivative of maltohexose at the anomeric carbon and showed its effectiveness in fluorescence and PET imaging of bacterial infections in rats, respectively ${ }^{32}$. Recently, Pang and co-workers ${ }^{33}$ developed theranostic nanoparticles loaded with purpurin 18 and targeted to bacteria by surface functionalization to maltohexose. These particles showed great potential in treating bacteria using sonodynamic therapy and assessed the specificity of their particles to the infection site using FLI imaging and showed an example of PAI using their particles. In parallel, our lab has developed an ${ }^{18} \mathrm{~F}-6$ " labeled maltose and maltotriose derivatives and showed their effectiveness in imaging bacterial infections through PET imaging ${ }^{34-36}$. While both imaging agents were specifically taken up in bacterial infections, the maltotriose derivative showed superior clearance from organs and better pharmacokinetic properties. Hence, we decided to utilize maltotriose as a scaffold for a photoacoustic and FLI agent of bacterial infections.

In this work, we report the development and evaluation of a derivative of maltotriose for photoacoustic and fluorescent 

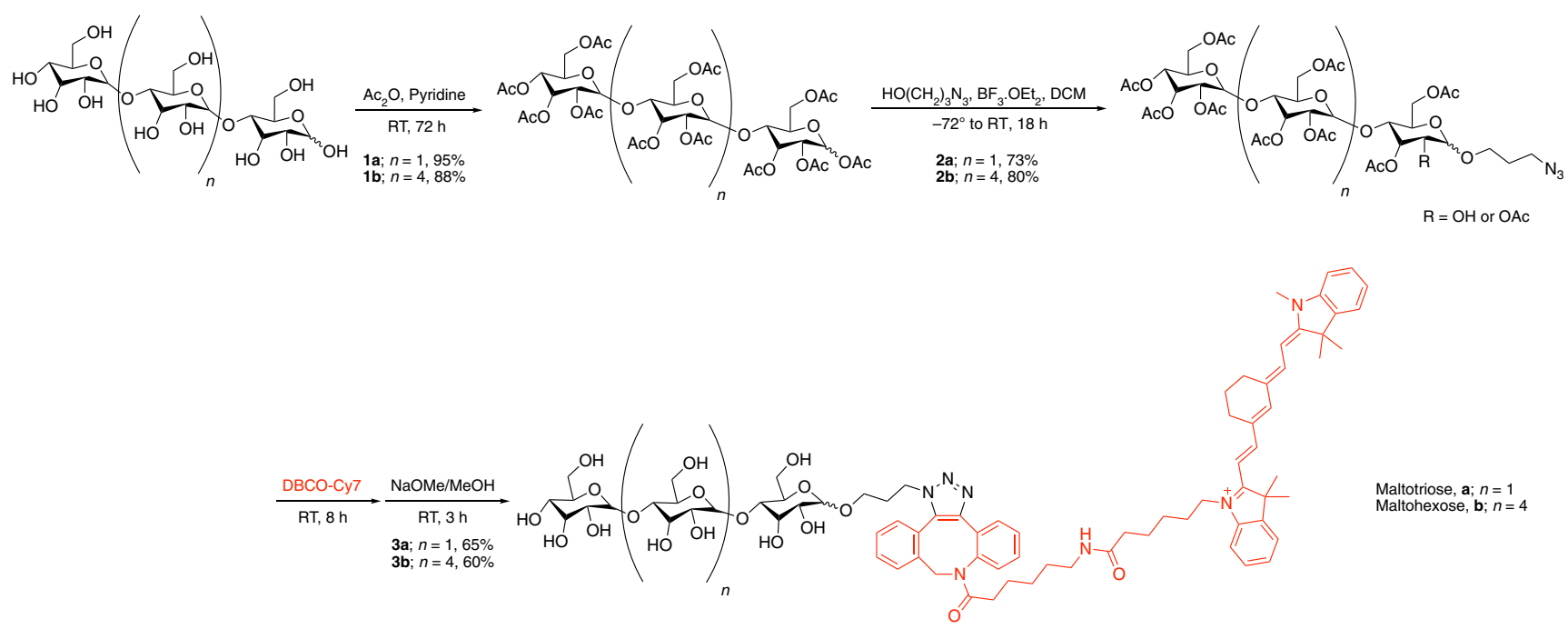

Fig. 1 Synthesis of Cy7-1-maltotriose and Cy7-1-maltohexose. $D B C O$ dibenzoyl cyclooctyne, $R T$ room temperature, $M e \mathrm{CH}_{3}, E t \mathrm{CH}_{3} \mathrm{CH}_{2}, D C M$ dichloro methane.

imaging of bacterial infections. The fluorescent dye is attached to the anomeric carbon (reducing end) of maltotriose, which is shown to have a smaller effect on the internalization of the sugar than functionalization at the $6 "$-position (non-reducing end). In addition, we conduct a face to face comparison study between maltotriose and maltohexose to determine the optimum scaffold for an imaging agent targeting the maltodextrin transporter.

\section{Results}

Synthesis of the fluorescent probes. Detailed synthesis of the fluorescent probes is provided in Supplementary Methods. Briefly, azide-functionalized maltotriose intermediate at the anomeric carbon (compound $\mathbf{2 a}$ ) was synthesized to allow ease of functionalization with a variety of signaling agents using copperfree click chemistry. Compound 1a was synthesized utilizing an adapted procedure $\mathrm{e}^{30,37}$ and collected as a colorless precipitate in $94 \%$ yield. Compound $\mathbf{2 a}$ was then produced from 1a using an adapted procedure ${ }^{37}$ resulting in a mixture of compound $2 \mathbf{a}$ as well as partially deacetylated 2a (Fig. 1, Supplementary Figs. 1 and 2 ) which was also previously reported ${ }^{37}$. Since the glycosylation was successful and the final product was going to be fully deacetylated, the reaction was carried on the mixture. The mixture was then functionalized with a commercially available fluorescent dye coupled to dibenzoyl cyclooctyne (Cy7-DBCO), through strain-promoted azide-alkyne [3+2] cycloaddition reaction. Sodium methoxide was then added to the crude mixture to deprotect the acetate groups before purification by reverse phase high-performance liquid chromatography (HPLC) to afford compound 3a in 65\% yield (Supplementary Fig. 3). In addition, the $\mathrm{Cy} 7$ derivative of maltohexose was prepared following the same synthetic route producing compound $3 \mathbf{b}$ in $60 \%$ yield (Fig. 1, Supplementary Fig. 4).

In vitro evaluation. A competition binding assay between the synthesized derivatives and ${ }^{3} \mathrm{H}$-maltose, which is taken up in bacteria through the maltodextrin transporter, was conducted. A significant reduction in ${ }^{3} \mathrm{H}$-maltose uptake in E. coli was observed when pre-incubated with azide- or Cy7-functionalized maltotriose or maltohexose (Fig. 2a, $P=0.0002, n=3$ per study). Furthermore, addition of the azide moiety and Cy7 functional groups on the anomeric carbon of maltotriose or maltohexose did not show any effect on its ability to block the uptake of ${ }^{3} \mathrm{H}$ maltose.
A direct assessment of the ability of Cy7-1-maltotriose (3a) and Cy7-1-maltohexose (3b) to be specifically taken up by a variety of bacterial strains containing the maltodextrin transporter was also conducted. Figure $2 \mathrm{~b}$ showcased the ability of this probe to be taken up by E. coli, Staphylococcus aureus, Bacillus subtilis, and Pseudomonas aeruginosa. Control studies, where azide-inactivated E. coli or E. coli mutations lacking components of the maltodextrin transporter were also evaluated and showed minimal uptake (Fig. 2b maroon, $P<0.0001, n=3$ per study).

In vivo evaluation in $E$. coli-induced myositis murine model. Cy7-1-maltotriose was evaluated in vivo in an E. coli-induced myositis murine model. Fluorescence images of the mice over time showed rapid accumulation of Cy7-1-maltotriose in the right thigh of the mice that was infected with $E$. coli. Such accumulation was not observed in the left thigh of the mice that was injected with heat-inactivated E. coli (Fig. 3a). In addition, significantly higher signal intensity in the right thigh compared to the left thigh starting at the $1 \mathrm{~h}$ imaging time point was observed $(P<0.0310, n=3)$, and the signal difference increased over time (Fig. 3b).

The same animal model was then used to monitor the infection site with PAI. As a control, photoacoustic images of both infected and control thigh muscle were collected before and after probe injection. Qualitatively, a higher photoacoustic signal in the infected thigh was observed when imaged after probe injection compared to that of the control muscle (Fig. 3c, Supplementary Fig. 11). In addition, quantitative analysis of the photoacoustic signal showed $\sim 3$-fold higher PA signal in the post-probe injection images of the infected thigh muscle compared to that before injection or to control thigh muscle $(P=0.0004$ and $P=$ 0.0002 , respectively) (Fig. $3 \mathrm{~d}$ ).

In the same animal model, a comparison study between the maltotriose and maltohexose derivatives (compound $\mathbf{3 a}$ and $\mathbf{3} \mathbf{b}$, respectively) was conducted. A higher fluorescence signal in the infected thigh (right) was observed in the mice injected with compound 3a (Fig. 4a, top). In addition, fluorescence signal in the infected muscle was quantified and normalized to control muscle (Fig. 4b) and showed $\sim 1.5$ times higher fluorescence signal in the infected thigh of mice injected with Cy7-1-maltotriose compared to those injected with Cy7-1-maltohexose. In PAI, significantly higher PA intensity in the infected thigh compared to control thigh was observed using either probes $(P<0.0013)$ (Fig. $4 \mathrm{c}, \mathrm{d}$, Supplementary Fig. 12). Slightly higher PA intensity in the 
a
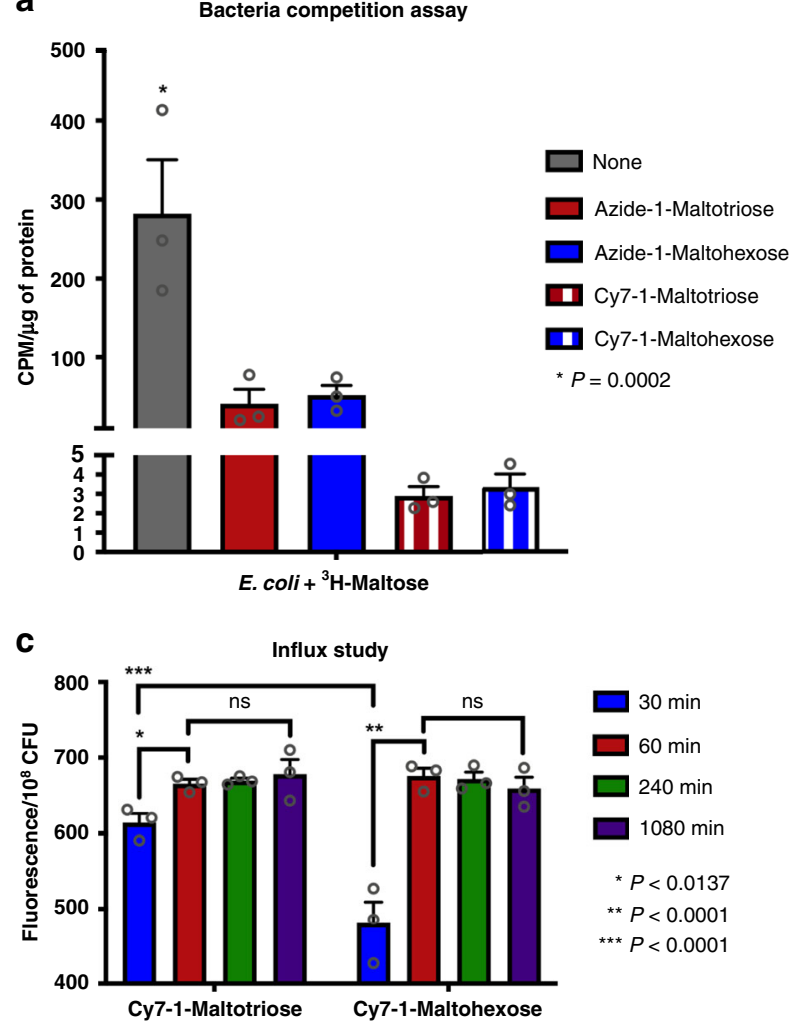

b

Bacteria binding assay

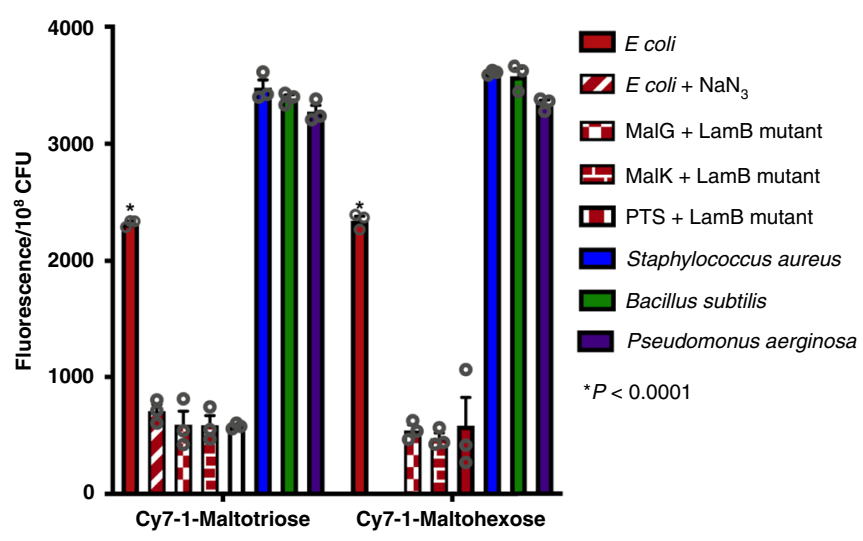

Fig. 2 In vitro evaluation of Cy7-1-maltotriose and Cy7-1-maltohexose. a Bar plot representation showing the effectiveness of 1-maltotriose and 1maltohexose derivatives in competing with the uptake of ${ }^{3} \mathrm{H}$-maltose in $\mathrm{E}$. coli. Significant reduction in ${ }^{3} \mathrm{H}$-maltose uptake in $E$. coli was observed with prior incubation with any of the 1-maltotriose or 1-maltohexose derivatives compared to $E$. coli incubated with only ${ }^{3} \mathrm{H}$-maltose $(P=0.0002, n=3)$. Data are presented as counts per minute (CPM) in each sample normalized to protein content ( $\mu$ g of protein). b Bar plot representation showing quantified fluorescence signal in E. coli, Staphylococcus aureus, Bacillus subtilis, and Pseudomonas aeruginosa after incubation with Cy7-1-maltotriose or Cy7-1maltohexose. As a control, sodium azide-inactivated $E$. coli, and $E$. coli mutants lacking components of the maltodextrin transporter were tested. Significant reduction in Cy7-1-maltotriose uptake in inactivated E. coli and E. coli mutations was observed $(P<0.0001, n=3)$. Significant reduction in Cy7-1maltohexose uptake in $E$. coli mutations was also observed $(P<0.0001, n=3)$. No significant difference in uptake between Cy7-1-maltotriose and Cy7-1maltohexose in all bacteria strains was observed $(P>0.05, n=3)$. c Bar plot representation of influx of Cy7-1-maltotriose and Cy7-1-maltohexose in $E$. coli overtime. Quantified fluorescence signal from influx study showcases significant increase in uptake of the probes when incubated for 60 min compared to 30 min incubation for both probes $(P<0.0137$ and $P<0.0001$ for $C y 7-1$-maltotriose and Cy7-1-maltohexose, respectively, $n=3)$. In addition, significantly higher fluorescence uptake of Cy7-1-maltotriose was observed compared to Cy7-1-maltohexose when incubated for 30 min was observed $(P<0.0001, n=$ 3). Bar graphs show mean and S.E.M. Statistical analysis was performed using one- and two-way ANOVA. The source data underlying Fig. $2 a-c$ are provided in a Source Data file.

infected thigh of mice injected with Cy7-1-maltotriose $(n=6)$ was observed compared to ones injected with Cy7-1-maltohexose $(n=3)$, but this difference was not significant (Fig. $4 \mathrm{~d}$, left, $P=$ 0.7571). However, the PA signal ratio between infected and control muscle was significantly higher for Cy7-1-maltotriose compared to Cy7-1-maltohexose (Fig. 4 d, right, $P=0.0353$ ).

To further assess the specificity of Cy7-1-maltotriose to bacteria containing the maltodextrin transporter, a similar study where a MalG + LamB mutant of E. coli was injected instead in the left thigh. In vivo FLI at 3 and $20 \mathrm{~h}$ post-injection also shows a rapid and significantly higher accumulation of Cy7-1-maltotriose in the E. coli-infected thigh (right thigh muscle) compared to the left thigh infected with $E$. coli mutation $(n=5$, Supplementary Fig. 13, $P<0.0001)$.

In vitro imaging of biomaterial infection. To examine the ability of our probe to detect bacteria on biomaterials, sterilized catheters were incubated in a solution of $10^{6} \mathrm{CFU}$ of $S$. aureus before incubation in a solution of Cy7-1-maltotriose. Evident FLI and BLI signals were observed on the catheters which were incubated with $S$. aureus solution followed by Cy7-1-maltotriose (Fig. 5a, left). In addition, minimal fluorescence signal in the control catheters which were not incubated with $S$. aureus was observed highlighting the low levels of nonspecific binding of Cy7-1-maltotriose to the catheter (Fig. 5a, b; $P<0.0001$ ).

In addition, the catheters which were only incubated with $S$. aureus solution, only showed the BLI signal and no fluorescence signal (Fig. 5a, right). The axial photoacoustic images showed noticeable photoacoustic signals on the surface of catheters incubated with both the bacteria and Cy7-1-maltotriose (Fig. 5c, left). While significantly lower photoacoustic signal was quantified on sterile catheters incubated only with Cy7-1-maltotriose (Fig. 5d; $P<0.0248$ ).

In vivo evaluation in $S$. aureus-infected wound murine model. In order to mimic a clinically relevant wound infection model, a bioluminescent strain of $S$. aureus (Xen 36) was inoculated into a superficial wound made on the back of the mouse. After confirmation of the presence of bacteria through BLI imaging, FLI and PAI were conducted (Fig. 6, before treatment). Mice were 
a

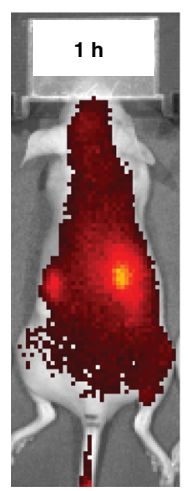

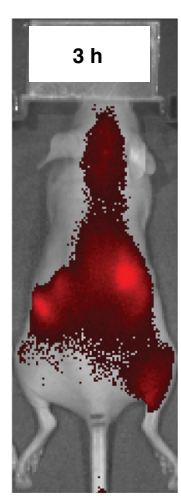

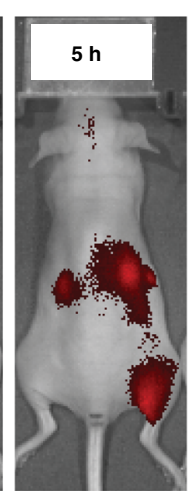

$3 \mathrm{~cm}$

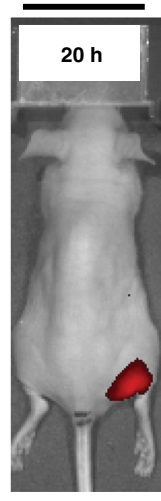

Epi-Fluorescence 10.0

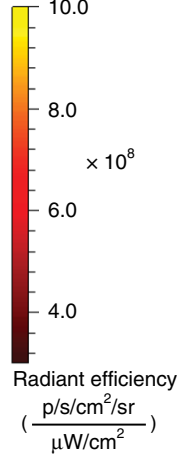

C

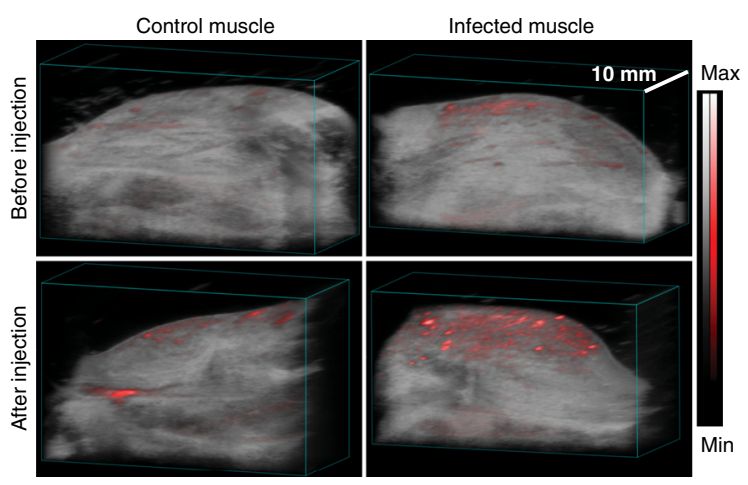

b

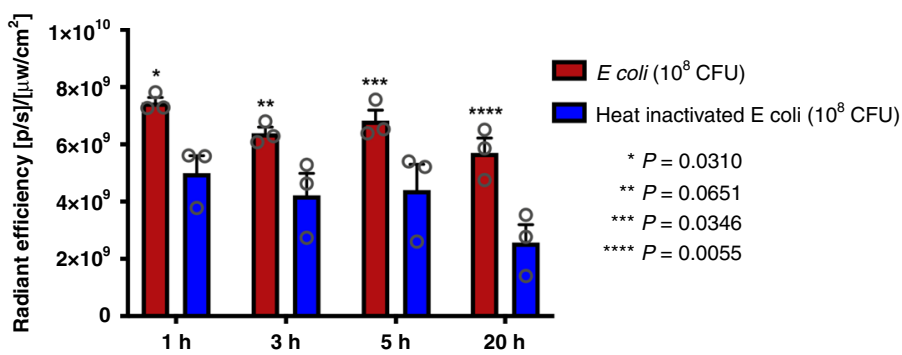

d

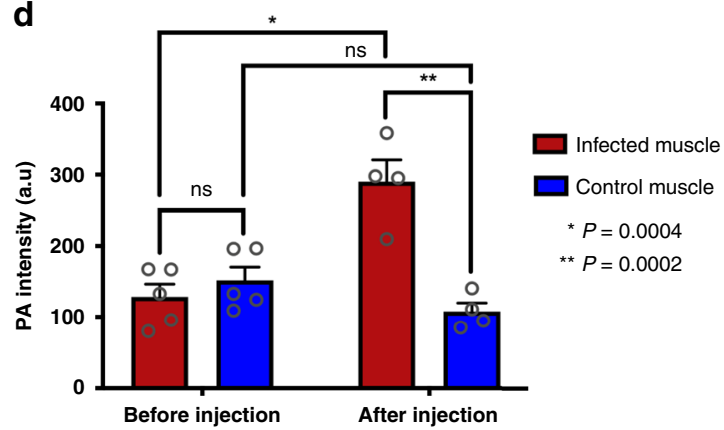

Fig. 3 In vivo validation of Cy7-1-maltotriose in an $\mathbf{E}$. coli-induced myositis murine model. a Fluorescence imaging shows accumulation of Cy7-1maltotriose in E. coli-infected thigh muscle as early as $1 \mathrm{~h}$ post systemic injection (right thigh muscle). No evident accumulation of the agent in thigh muscle injected with $10^{8}$ CFUs of heat-inactivated E. coli (left thigh muscle). b Bar plot representation of quantified fluorescence signal in right and left thigh muscle overtime. As early as $1 \mathrm{~h}$ post-injection, a significantly higher fluorescence signal was found in muscle infected with $E$. coli (right thigh) compared to muscle infected with heat-inactivated $E$. coli (left thigh) $(P=0.0310, n=3)$. c 3D rendered photoacoustic image overlaid on ultrasound image of a mouse's left (control) and right (infected) thigh muscle before and $20 \mathrm{~h}$ post-injection of Cy7-1-maltotriose. Qualitatively, the image of the infected thigh muscle (bottom right) shows higher photoacoustic signal relative to image of the same muscle acquired before probe injection (top right) and image of the control thigh muscle (bottom left). d Bar plot representation of the quantified photoacoustic signal intensity in the photoacoustic images acquired before and $20 \mathrm{~h}$ after injection of Cy7-1-maltotriose. The quantified signal of the infected thigh muscle was significantly higher post-injection of the probe compared to that before injection ( $n=4$ and 5 respectively, $P=0.0004$ ). In addition, the post-injection signal in the infected thigh muscle was significantly higher than that of the control muscle $(n=4, P=0.0002)$. Bar graphs show mean and S.E.M. Statistical analysis was performed using two-way ANOVA. ns no statistically significant difference $(P>0.05)$. The source data underlying Fig. 3b and $d$ are provided in a Source Data file.

then divided into two groups where one was administered subcutaneously a therapeutic dose of vancomycin twice daily (Treated group, $n=5$ ), while the other group was not treated with vancomycin (untreated group, $n=4$ ). After 7 days of antibiotic treatment, Cy7-1-maltotriose was administered and imaging completed $20 \mathrm{~h}$ post-injection. No BLI nor FLI signal and minimal PAI signal in the wound were observed in the treated group post-treatment (Fig. 6a, b, Treated group-After). While the untreated group showed evident BLI, FLI, and PAI signal in the wound. In addition, a significant decrease in the fluorescence and photoacoustic signal $\left(4.33 \pm 0.96 \times 10^{8}\right.$ vs $1.48 \pm 0.15 \times 10^{8}$ radiance efficiency and $0.99 \pm 0.09$ vs $0.37 \pm 0.06$ a.u. respectively, $P<0.0001, n=4)$ was observed in the images collected posttreatment compared to before treatment (Fig. 6c, d).

In a similar animal model, different amounts of the bioluminescent Xen 36 strain Staphylococcus aureus $\left(10^{4}, 10^{6}\right.$, or $10^{8} \mathrm{CFU}$; $n=3,3$, and 5 respectively) were inoculated subcutaneously in a small wound formed on the back of the mice. FLI images $18 \mathrm{~h}$ postinjection showed accumulation of Cy7-1-maltotriose in the wound in all three mice groups where the location of the FLI signal directly correlated to that of the BLI signal (Fig. 7a). In addition, a significant increase in the quantified fluorescence signal was observed with an increase in quantified BLI signal (i.e. increase in CFU in the wound) (Fig. 7b).

\section{Discussion}

After evaluating ${ }^{18} \mathrm{~F}$-labeled analogs of maltose and maltotriose, our group demonstrated that an ${ }^{18} \mathrm{~F}-6$ "-labeled maltotriose showed the most optimal pharmacokinetic and pharmacodynamic properties to specifically image bacterial infections ${ }^{36}$. The radio-label, in this case, was at the non-reducing end of the molecule and the addition of ${ }^{18} \mathrm{~F}$ did not interfere with its internalization through the maltodextrin transporter ${ }^{36}$. While the PET tracer would have extensive applications in a hospital setting, an optical imaging approach would have advantages in an outpatient setting or in the ER for the local evaluation of suspected sites of infection. PAI has the added advantage of increased depth penetration, translation into ultrasound-based settings and the ability to provide valuable real-time anatomical information. We therefore set out to develop a photoacoustic version of the previously reported maltotriose imaging agent. Unfortunately, the ability of the azide-functionalized maltotriose to block ${ }^{3} \mathrm{H}$ maltose uptake in E. coli dropped when the azide was functionalized at the 6" position (Azide-6"-Maltotriose) compared to the 1 position (Azide-1-Maltotriose) (Supplementary Fig. 5). These results confirm previous reports stating the adverse effects of functionalization of the non-reducing end of the maltodextrin on its binding affinity to maltose-binding protein (MBP) which can severely compromise internalization through the maltodextrin 
a
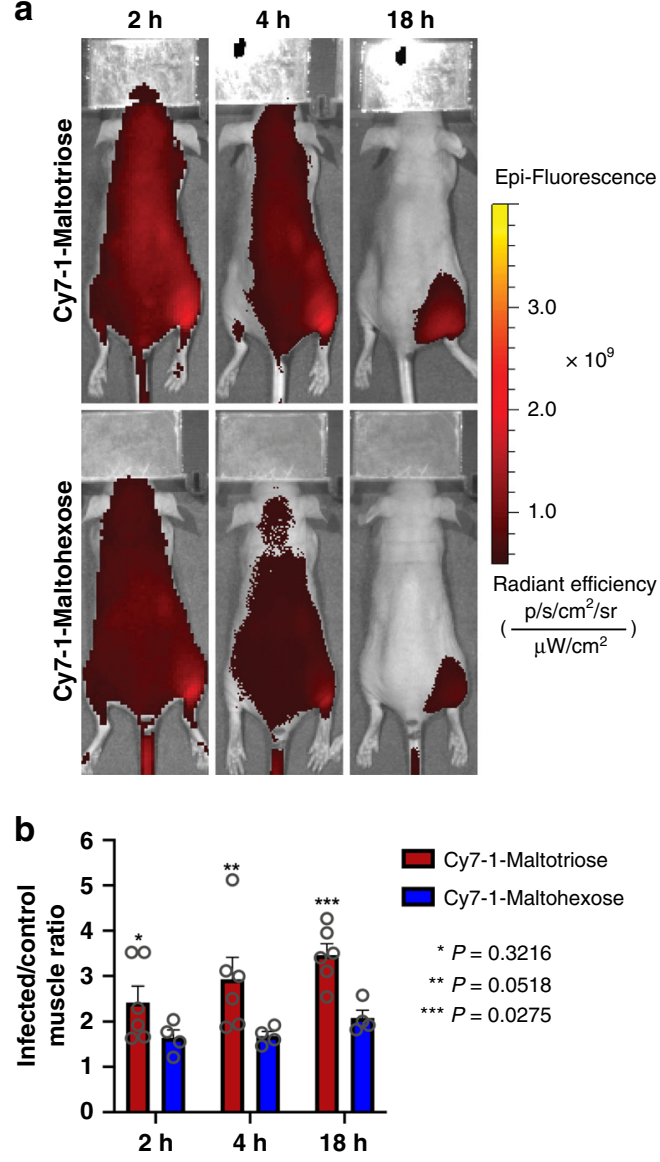

C

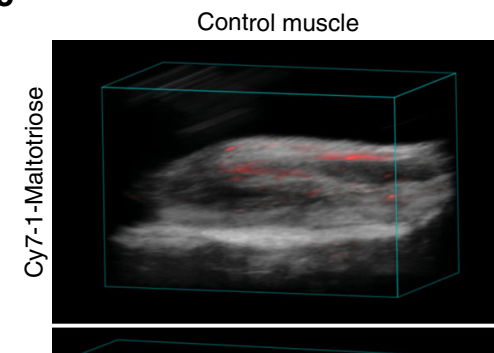

Control muscle

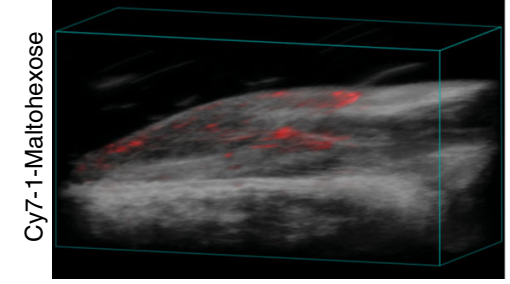

d

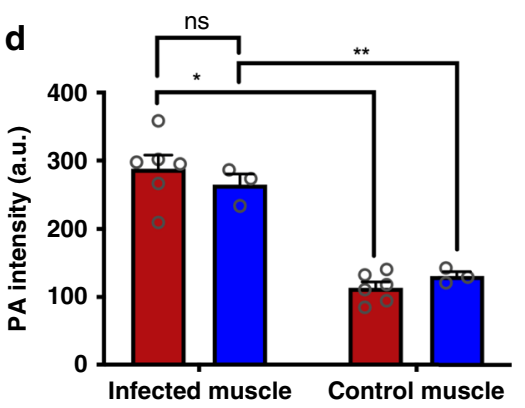

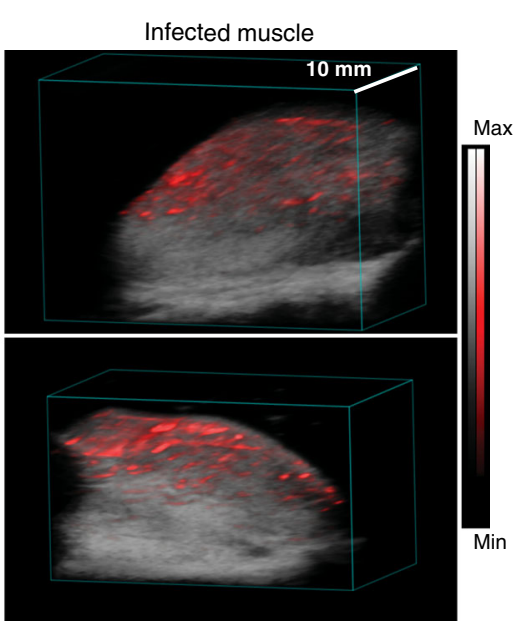

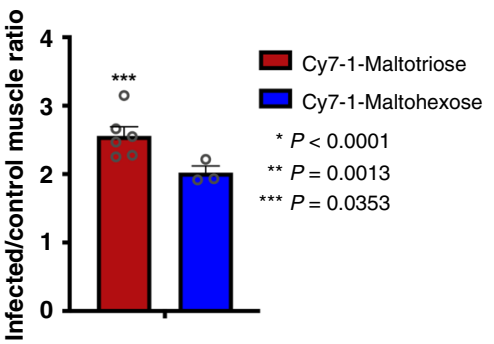

Fig. 4 In vivo comparison between Cy7-1-maltotriose and Cy7-1-maltohexose in an E. coli-induced myositis murine model. a In vivo fluorescence images at 2, 4, and $18 \mathrm{~h}$ post-injection of either Cy7-1-maltotriose (top) or Cy7-1-maltohexose (bottom) showing accumulation of both probes in the right thigh muscle (E. coli). Qualitatively higher fluorescence signal in the infected muscle (right thigh) when injecting Cy7-1-maltotriose (top) compared to Cy7-1maltohexose (bottom) is observed. $\mathbf{b}$ Bar plot representation of ratio of fluorescence in the infected thigh vs control thigh. Higher fluorescence signal in the infected muscle (right thigh) when injecting Cy7-1-maltotriose $(n=6)$ was observed compared to injecting Cy7-1-maltohexose $(n=4)$ and this was significant at $18 \mathrm{~h}$ post-injection $(P<0.0275)$. c 3D rendered photoacoustic image overlaid on ultrasound image of a mouse $21 \mathrm{~h}$ post-injection of Cy7-1maltotriose (top) and Cy7-1-maltohexose (bottom). Images show infected (right thigh) and control (left thigh) muscle. Evident PA signal is observed in the infected thigh muscle when injecting either compounds while minimal signal is observed in the control thigh muscle. $\mathbf{d}$ Bar plot representation of the quantified photoacoustic signal intensity in the photoacoustic images acquired $21 \mathrm{~h}$ after injection of Cy7-1-maltotriose and Cy7-1-maltohexose. The quantified signal shows significantly higher PA signal in the infected muscle compared to control muscle when injecting either compounds (left). No significant difference in PA signal was observed between the two compounds. Significantly higher infected over the control PA signal ratio was observed when injecting Cy7-1-maltotriose $(n=6)$ compared to Cy7-1-maltohexose $(n=3)(P<0.0353)$ (right). Bar graphs show mean and S.E.M. Statistical analysis was performed using two-way ANOVA. ns no statistically significant difference $(P>0.05)$. The source data underlying Fig. $4 \mathrm{~b}$ and $\mathrm{d}$ are provided in a Source Data file.

transporter ${ }^{38}$. Ning and co-workers ${ }^{30}$ previously reported the development of a fluorescent derivative of maltohexose which was functionalized at the reducing end and showed promising results in optical imaging of bacterial infections. However, Axer et al. ${ }^{38}$ reported that maltotriose could potentially provide a more optimal scaffold in bacterial infection imaging agents compared to other maltodextrins (e.g. maltohexose). This led us to believe that a fluorescent derivative of maltotriose can potentially be a superior bacterial infection imaging agent when functionalized at the reducing end. Accordingly, we sought to functionalize the dye at the anomeric side (reducing end) of maltotriose and decided to also synthesize the maltohexose derivative to properly identify the optimal scaffold for our bacterial infection imaging agent.

We have established a modified synthetic route that produces an azide-1-functionalized maltotriose or maltohexose using an adapted glycosylation procedure ${ }^{37}$ in two high yielding steps
(Fig. 1). Developing such an intermediate simplifies the functionalization of the maltotriose and maltohexose scaffold to a variety of signaling agents using copper-free click chemistry or copper catalyzed azide-alkyne reaction. In this work, Cy7 dye was chosen due to its great photo and chemical stability and linked to maltotriose $(n=1)$ or maltohexose $(n=4)$ through azide-DBCO copper-free click chemistry (Fig. 1).

Following synthesis of the probes, it was essential to assess the effects of the azide and Cy7 motifs on the ability of maltotriose and maltohexose to internalize into bacteria. This was assessed by running a competition assay between the tested derivatives and ${ }^{3} \mathrm{H}$-maltose as well as uptake studies (Fig. 2a and b, respectively). Both probes were taken up in a wide variety of Gram-positive and Gram-negative bacterial strains (Fig. 2b). The specificity of the probes for imaging live bacteria that contain the maltodextrin transporter was demonstrated in uptake studies 
a

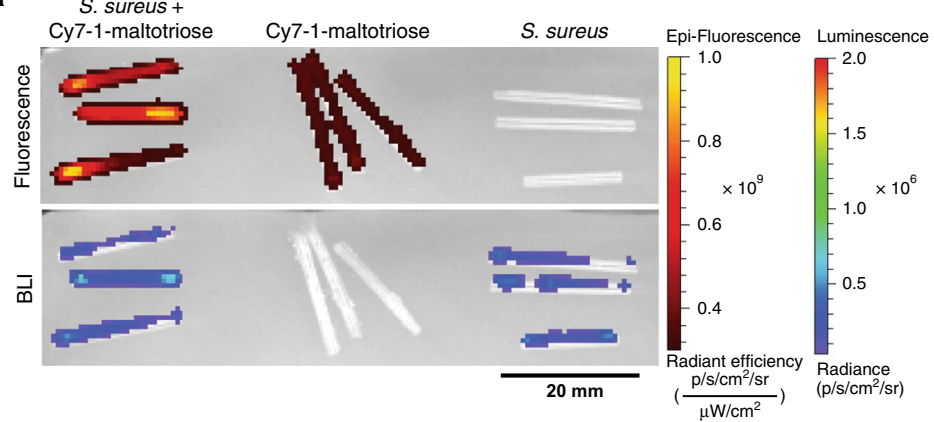

C

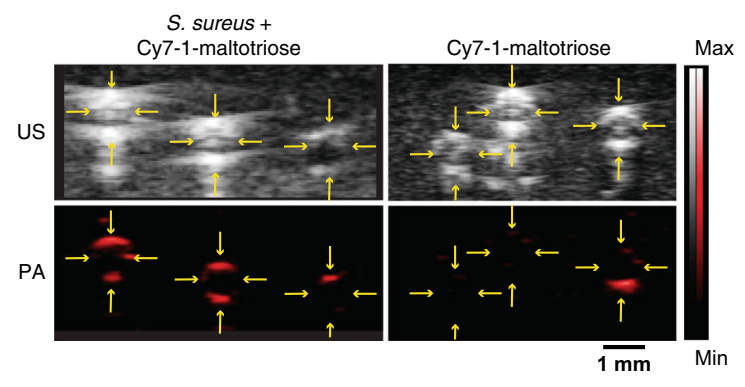

b

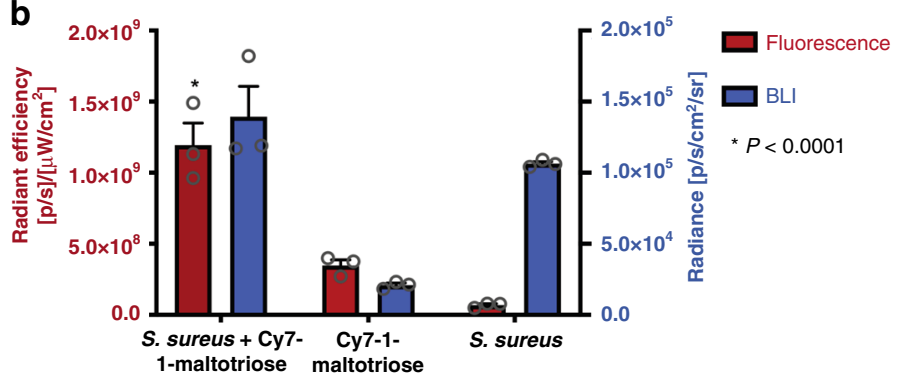

d

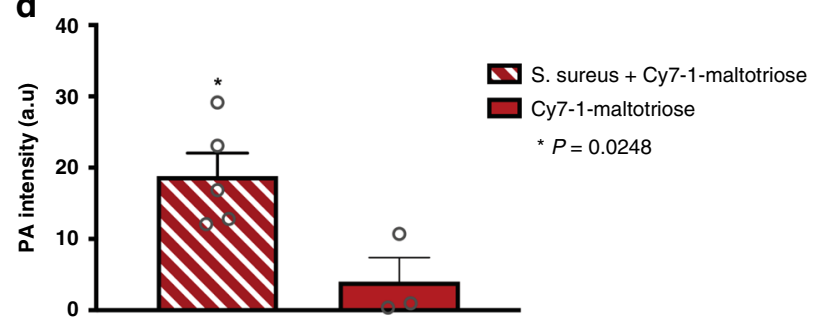

Fig. 5 In vitro evaluation of Cy7-1-maltotriose in a S. aureus-infected biomaterial model. a FLI and BLI images showcasing the presence and lack of S. aureus on catheters. High FLI and BLI signals were observed in catheters that were incubated with S. aureus followed by Cy7-1-maltotriose (left) compared to sterile catheters that were only incubated with Cy7-1-maltotriose (middle). Similarly, no FLI signal and only BLI signal was observed on catheters that were incubated with S. aureus only (right). b Total FLI and BLI signals in catheters infected with bioluminescent S. aureus and incubated with Cy7-1-maltotriose. Fluorescence quantification was plotted to the left $y$-axes (red) while BLI signal plotted to the right $y$-axes (blue) and showed significantly higher FLI signal in infected catheters compared to sterile catheters post incubation with a solution of Cy7-1-maltotriose $(P<0.0001, n=3)$. c Axial US and PA imaging showcasing the presence and lack of $S$. aureus on catheters upon incubation with Cy7-1-maltotriose. An evident PA signal was observed in axial images of catheters incubated with S. aureus followed by Cy7-1-maltotriose (bottom left), compared to sterile catheters that were only incubated with Cy7-1-maltotriose (bottom right). Yellow arrows mark the catheter's outline. $\mathbf{d}$ Bar plot representation of the quantified photoacoustic signal intensity in the axial photoacoustic images of infected and sterile catheters post incubation with Cy7-1-maltotriose. The infected catheters $(n=5)$ had significantly higher PA signal compared to sterile catheters $(n=3)$ post incubation with Cy7-1-maltotriose $(P=0.0248)$. Bar graphs show mean and S.E.M. Statistical analysis was performed using two-way ANOVA. The source data underlying Fig. $5 b$ and $d$ are provided in a Source Data file.

with azide-inactivated $E$. coli and $E$. coli mutants that lack components of the maltodextrin transporter $(P<0.0001, n=3)$ (Fig. 2b, maroon).

Preclinical evaluation of Cy7-1-maltotriose in E. coli-induced myositis murine model using FLI and PAI was conducted. FLI was used as the primary tool to assess the specificity and uptake kinetics of the probe in bacterial infections because it allows whole-mouse imaging and provides information that can be compared to previous optical imaging agents for infection. The kinetics of accumulation of the probe were examined and illustrate rapid clearance of Cy7-1-maltotriose through the kidneys. Notably, specific signal accumulation at the site of the infected muscle occurred very rapidly (as early as $1 \mathrm{~h}$ ), again illustrating the potential utility in field hospitals where quick decisions need to be made (Fig. 3a, b). In addition, photoacoustic images were also able to show a significant difference between the infected and control muscle (Fig. 3c, d, Supplementary Fig. 11). We believe PAI will have several advantages over FLI, as we have discussed in the introduction particularly the enhanced imaging penetration depth. Another potential advantage lies with the instrumentation rapidly being developed ${ }^{39,40}$, and the ease with which these handheld scanners can be integrated into existing ultrasound systems ${ }^{41}$.

It was then important to identify the optimum maltodextrin scaffold to utilize in our bacterial infection imaging agent. A copious amount of work was conducted to assess the difference in internalization kinetics, stability, and retention between maltodextrins in a variety of bacterial strains ${ }^{38,42-48}$. We conducted a comparison between the maltotriose and maltohexose analogs of our imaging agent. Fluorescent maltohexose was previously used by Ning and co-workers ${ }^{30,32}$ as a fluorescent imaging agent for bacterial infection and showed specificity to bacterial infections in rat models. In vitro competitive and uptake studies looked similar to that of Cy7-1-malotriose (Fig. 2a, b, respectively). A comparison study was then conducted in the E. coliinduced myositis murine model. These studies demonstrated the pharmacokinetic advantages of maltotriose over maltohexose in vivo. As shown in Fig. 4, both Cy7-1-maltotriose and Cy7-1maltohexose showed specific uptake in the infected muscle (right thigh) which is distinguishable from the control muscle (left thigh) (Fig. 4a, b). Eighteen hours post-injection, Cy7-1maltotriose had 2.6-fold higher fluorescence compared to that of Cy7-1-maltohexose $\left(15.5 \pm 5.0 \times 10^{9}\right.$ and $6.02 \pm 0.5 \times 10^{9}$ radiance efficiency, respectively; $P<0.0275, n=6$ and 4 , respectively). This can be attributed to the faster clearance of the Cy7-1maltohexose as compared to Cy7-1-maltotriose from circulation due to higher hydrophilicity $(\mathrm{C} \log \mathrm{P}=-5.8$ vs -12.3 for maltotriose and maltohexose, respectively). The in vivo studies match the observations made in our in vitro influx studies where a much faster uptake was observed for the maltotriose derivative (Fig. 2c, $P<0.0001)$.

Stability studies on both compounds in murine, rat, and human plasma as well as phosphate-buffered saline (PBS) over 
a

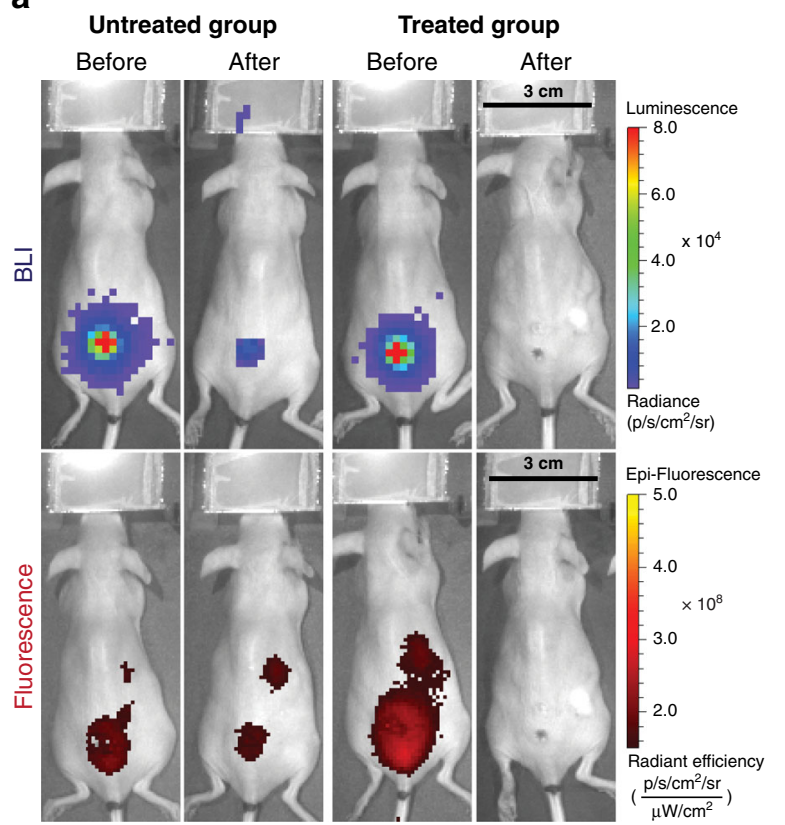

b

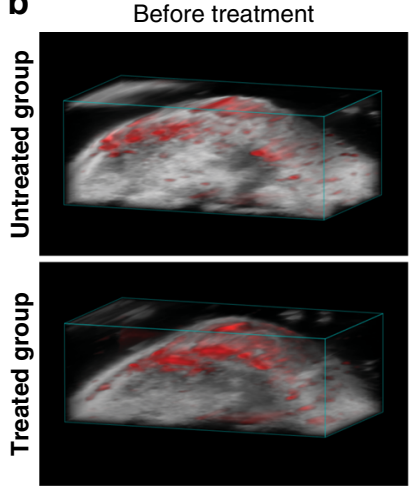

C

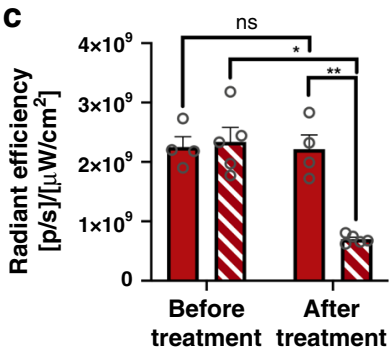

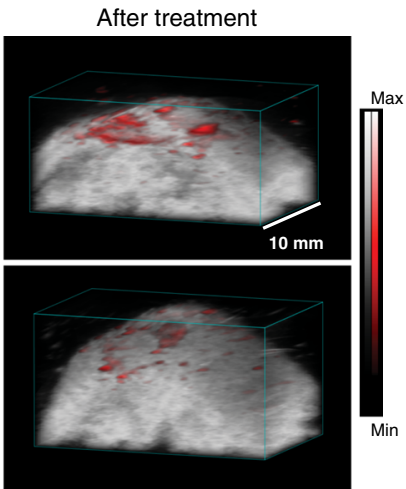

d

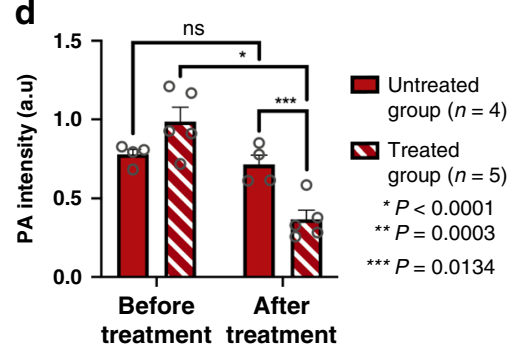

Fig. 6 In vivo evaluation of Cy7-1-maltotriose in a S. aureus wound infection murine model. a BLI and FLI images of mice with a wound infected with $10^{6}$ CFUs of bioluminescent S. aureus $19 \mathrm{~h}$ post-injection of Cy7-1-maltotriose without (Untreated Group) and with (Treated Group) treatment with vancomycin for 7 days and before and after treatment. FLI (bottom-Before) shows accumulation of the probe in the S. aureus located by BLI (top-Before). In the untreated group (left panel) both BLI and FLI showcases presence of S. aureus infection in the wound after 7 days (Untreated Group-After). While in the treated group (right panel), complete disappearance of S. aureus infection was observed in the FLI image and confirmed by BLI (Treated Group-After). b 3D rendered PA image overlaid on US image of a mouse from Untreated (Top row) and Treated (Bottom row) groups before and after treatment. Images were acquired $20 \mathrm{~h}$ post-injection of Cy7-1-maltotriose. Similar to observations in FLI, the Treated group showed lower PA signal post-treatment (bottom right) than that before treatment (bottom left). c Total in vivo fluorescence signal in wound infected with $10^{6} \mathrm{CFUs}$ of bioluminescent $\mathrm{S}$. aureus $19 \mathrm{~h}$ after tail vein injection of Cy7-1-maltotriose. Significant reduction of FLI signal after treating the mice with vancomycin for 7 days in the treated group ( $n=5, P<$ 0.0001) was observed, while no difference was observed in the untreated group before and after 7 days $(n=4)$. $\mathbf{d}$ Bar plot representation of the quantified average PA signal intensity in the PA images acquired $20 \mathrm{~h}$ after injection of Cy7-1-maltotriose before and after antibiotic treatment. PA quantification data showed a similar trend to that obtained from FLI images. Bar graphs show mean and S.E.M. Statistical analysis was performed using two-way ANOVA. ns no statistically significant difference $(P>0.05)$. The source data underlying Fig. $6 \mathrm{c}$ and $\mathrm{d}$ are provided in a Source Data file.

time showed substantial differences in stability between the maltotriose and maltohexose derivatives. Specifically, Cy7-1maltohexose is shown to rapidly break down into what we hypothesize to be smaller sugar forms in mere minutes in plasma ( $<2 \%$ intact by $2 \mathrm{~h}$ in rat and murine and around $10 \%$ in humans, Supplementary Fig. 6a, right, Supplementary Fig. 7b). While around $70 \%$ of Cy7-1-maltotriose was intact in murine and rat after $2 \mathrm{~h}$ and no degradation of maltotriose in human plasma was observed (Supplementary Fig. 6a, left, Supplementary Fig. 7a). In addition, both maltotriose and maltohexose were stable in PBS for up to $24 \mathrm{~h}$ (Supplementary Figs. 6a and 7, right). These results directly correlate with previous reports by Axer et al. ${ }^{38}$. In the PA imaging study, slightly higher PA intensity in the infected thigh using Cy7-1-maltotriose $(n=6)$ was observed compared to Cy71-maltohexose $(n=3)$ ( 288.2 vs 264.6 a.u., respectively) yet this difference was not significant $(P=0.139)$. But when looking at the PA intensity ratio of infected over control muscle, a significantly higher ratio using the maltotriose derivative vs maltohexose was observed ( 2.5 vs 2 , respectively, $P=0.0353$ ). This further resembles the data shown in the FLI study and is most likely due to the lower sensitivity of PA in detecting Cy7 which is more geared for FLI rather than photoacoustic detection (Supplementary Figs. 8-10).

Evidently, the slower bacterial uptake of the maltohexose derivative, its faster clearance due to its hydrophilicity, and its lower plasma stability provide proof that Cy7-1-maltotriose is the superior bacterial-imaging probe. Further investigation into the exact mechanism of uptake of the two compounds, their metabolism and their ability to be taken up by metabolically inactive bacteria is necessary. In addition, there is some evidence in Enterococcus that maltotriose and maltohexose could be taken up by different transporters at different rates ${ }^{46}$. More experiments are underway such as using CRISPR-based knockouts of individual subunits of the two different transport systems to address this question.

We believe one potential application of Cy7-1-maltotriose is to detect and monitor the treatment of bacterial infections in susceptible sites (i.e. wounds, surgical sites, and medical implants). It is shown that device associated infections account for around $25.6 \%$ of all healthcare-associated infections in the United States ${ }^{6}$. For example, it is projected that the total number of knee arthroplasties (TKA) performed per year in the United States will be around three million by 2030 (refs. ${ }^{49,50}$ ). One of the leading reasons for TKA failure is due to periprosthetic joint infection (PJI) which can occur in $1-2 \%$ of the cases. Unfortunately, current PJI diagnostic tools necessitate sample collection from the prosthetic site and are divided into culture-based tools (ex. peri-implant tissue culture, synovial culture, and histology) $)^{51}$ and culture-independent tools (ex. Ibis PLEX-ID technology ${ }^{52}$, MALDI-TOF mass spectroscopy ${ }^{53}$, nextgeneration sequencing ${ }^{54}$ ). The latter tools have not yet been 

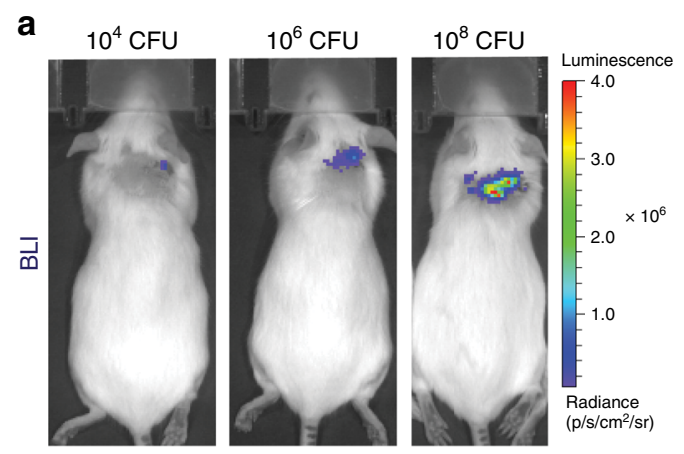

b
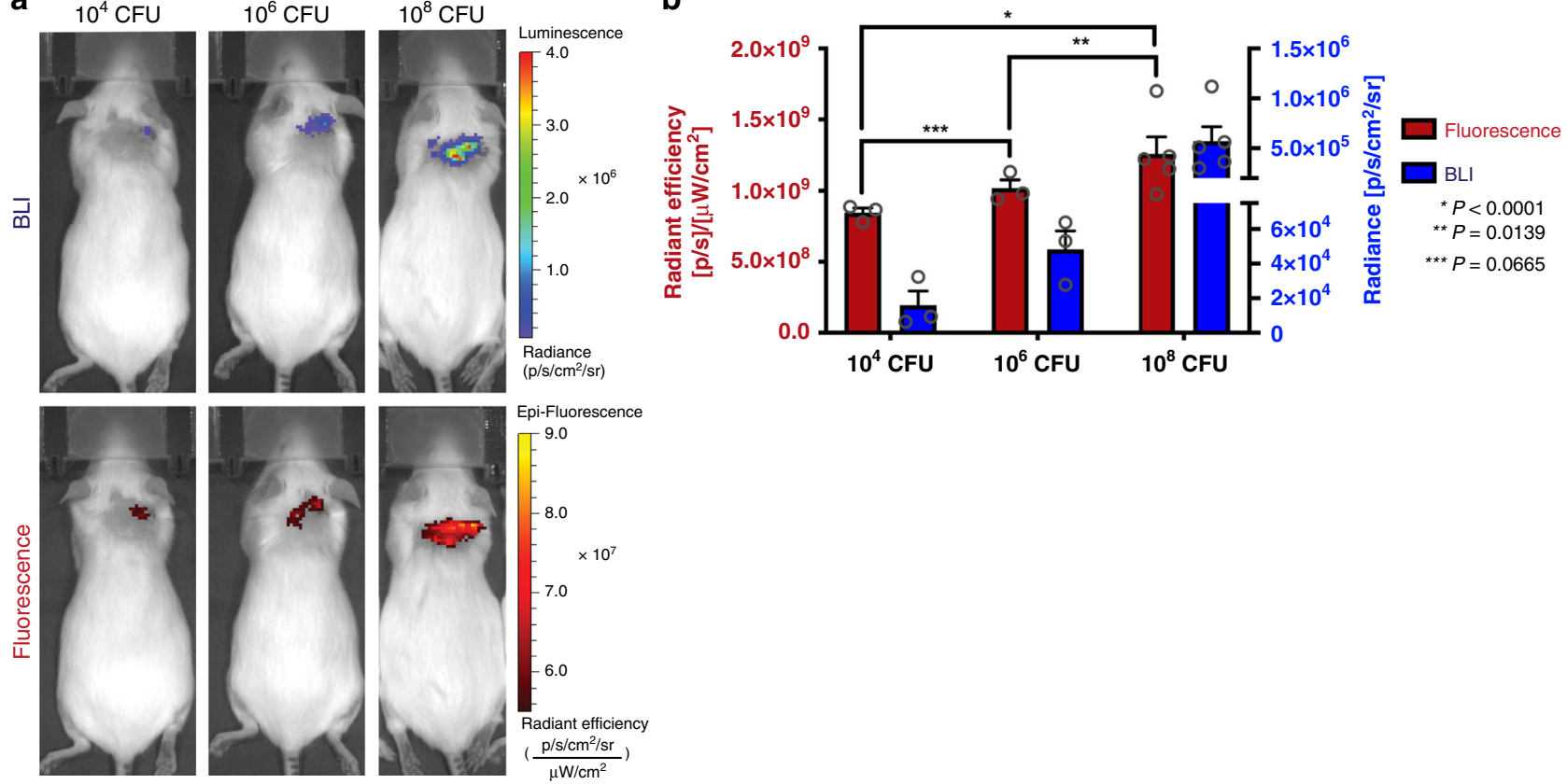

Fig. 7 In vivo evaluation of Cy7-1-maltotriose in a S. aureus wound infection murine model. a FLI and BLI of mice with a wound infected with different amounts of bioluminescent S. aureus $22 \mathrm{~h}$ post-injection of Cy7-1-maltotriose. FLI images (bottom) show accumulation of the probe in the S. aureus in the same area as indicated by BLI (top). b Total in vivo FLI and BLI signals in wound infected with $10^{4}, 10^{6}$, and $10^{8}$ CFUs of bioluminescent S. aureus ( $n=3,3$, and 5 , respectively) and $22 \mathrm{~h}$ after tail vein injection of Cy7-1-maltotriose. Fluorescence quantification was plotted to the left $y$-axes (red) while BLI signal plotted to the right $y$-axes (blue) and showed increase in FLI signal with increase in BLI signal $\left(r=0.928 ; r^{2}=0.8612\right)$. Bar graphs show mean and S.E.M. Statistical analysis was performed using two-way ANOVA. The source data underlying Fig. 7b are provided in a Source Data file.

adopted in the clinic due to high cost and lack of sufficient validation $^{55,56}$. Thus, a non-invasive tool for PJI diagnostic which does not rely on sampling from the site will be very valuable and allow differentiation from inflammation which is often confused with infections in these situations as they present with similar symptoms. Since $S$. aureus is the most prevalent pathogen in medical device infections and accounts for $\sim 32 \%$ of medical device infections ${ }^{6}$, we decided to assess $S$. aureus infections of biomaterials using PAI and FLI upon incubation with Cy7-1-maltotriose. As shown in Fig. 5, Cy7-1-maltotriose is able to differentiate infected from uninfected catheters both using FLI and PAI. This provides the possibility of using PAI to image implants particularly in the joints as well as fracture fixtures to screen for infections.

We then evaluated the ability of our imaging platform in assessing wound infections as well as determining the effectiveness of antibiotic treatment in vivo. S. aureus is used in this study since it is the most common cause of SSIs and results in a $5 \%$ increase in mortality ${ }^{8}$. Antibiotic treatment reduced the bacterial burden and the probe uptake reflected this decrease and showed significant differences in signal between treated and untreated groups in both FLI and PAI (Fig. 6). In addition, both FLI and PAI were able to distinguish between the treated and untreated groups (Fig. $6 \mathrm{c}, \mathrm{d}, P=0.0003$ and 0.0134 , respectively) illustrating the utility of this platform in assessing the effectiveness of treatment.

Further FLI evaluation showed an increase in FLI signal (i.e. Cy7-1-maltotriose accumulation) with an increase in CFU of $S$. aureus in the wound (i.e. increase in BLI signal). Wounds infected with as low as $10^{4}$ CFU were detectable by FLI of Cy7-1maltotriose and showed a strong correlation with the location of the BLI signal (Fig. 7a, b). In addition, similar imaging done with the $S$. aureus wound model showed that once injected, the probe remains at the infected wound for up to $144 \mathrm{~h}$ post-injection, which allows serial imaging without having to administer the probe repeatedly (Supplementary Fig. 14).

In summary, a maltotriose-based infection imaging agent functionalized with an optical dye at the anomeric carbon using copper-free click chemistry was developed. In vitro evaluation showcases the efficacy and specificity of the probe to be taken up by a variety of metabolically active Gram-positive and Gramnegative bacteria. In vivo assessments and in vitro stability studies showed superior performance of the maltotriose-based probe compared to its maltohexose analog. Molecular fluorescence and PAI of bacterial infection was demonstrated and showed the ability to specifically detect bacterial infections in myositis and wound infection models and on biomaterial. Evaluations in $S$. aureus-infected wound model showcased the ability of the probe to detect and differentiate between wounds infected with different amounts of CFUs as well as determine the effectiveness of vancomycin treatment. While the Cy7 dye used in this imaging agent possesses many great characteristics such as in vivo stability and optimum pharmacokinetics when bound to maltotriose, this dye is not approved for clinical use and is more suitable for FLI than PAI. This could explain the higher sensitivity in detecting this probe using fluorescence imaging compared to PAI (Supplementary Figs. 8-10). Hence, we are investigating attachment of a variety of clinically approved dyes as well as dyes more geared for PAI to further enhance its performance. More specifically, the dye needs to have great plasma stability, high photostability, low toxicity and ease of synthesis in a GMP facility. Furthermore, possessing more red-shifted as well as distinctive absorbance characteristics from intrinsic signals (such as hemoglobin and melanin) will further improve imaging depth and signal quantification, respectively. The substitution of the dye may affect the distribution of the imaging agent and a thorough in vivo evaluation of the new probes will be necessary. In addition, while 
whole-body PAI systems in humans do not exist, small-animal imaging systems have been reported and could provide better means to evaluate the novel agents in a preclinical setting 57,58 . We believe that we have identified the ideal scaffold for an infection imaging agent and with our established synthetic scheme, we can quickly synthesize and clinically translate these new probes.

While PAI is growing to be a promising diagnostic tool, it still possesses several limitations that are being addressed. Namely, the limited field of view and imaging depth constricts its utility in humans to localized imaging (no whole-body imaging). Similar to ultrasound imaging, this tool is highly dependent on the operator's experience and means to automate this technique will help overcome this issue and allow better and more accurate reproducibility of the images (e.g. Robotic handles and software tools to precisely delineate imaging plane for accurate reproducibility) ${ }^{16}$. Alternatively, three-dimensional (3D) volume scanning should also assist in reducing the dependence of the operator's experience. Furthermore, several hurdles related to accurate signal quantification (such as differentiating intrinsic signals from imaging agent, reduced accuracy with increased depth and effects of disease progression or tissue healing on PA signal intensity) need to be addressed to conduct molecular PAI of disease ${ }^{16}$. Such limitations can be somewhat mitigated by proper choice of the photoacoustic dye (as mentioned above), choosing the best excitation wavelengths that can assist in differentiating the signal (e.g. background subtraction), the use of models for fluence correction ${ }^{16}$ and other AI tools which can take into account any PA signal intensity changes occurring during structural changes caused by disease progression, inflammation, and healing.

Nevertheless, ultrasound imaging has been widely used to diagnose late-stage infections by visualizing the anatomical changes caused by the spread of infection ${ }^{9}$. Compact and easily transportable PAI systems are being produced with state of the art technologies by many research groups including our own ${ }^{16,41,59-61}$. This makes us believe that the translation of PA for infection imaging can be readily adapted with the pairing of the proper system and imaging agent ${ }^{16,41,59,60}$. The development of a maltotriose-based PA agent accompanied by rapid advances in PAI provides a readily available, non-invasive, cost-effective, real-time imaging tool that can enable early detection of bacterial infections during surgery and injury with high resolution.

\section{Methods}

Plasma stability studies. Compound $\mathbf{3 a}$ or $\mathbf{3 b}(100 \mu \mathrm{M})$ was incubated in either PBS (1×), murine, human, or rat plasma at $37^{\circ} \mathrm{C}$ for up to $24 \mathrm{~h}$. At each time point $(0,2,4,10$, and $24 \mathrm{~h})$, a $100 \mu \mathrm{L}$ aliquot of the mixture was taken and added to an ice-cold acetonitrile solution $(200 \mu \mathrm{L})$ and vortexed for $10 \mathrm{~s}$. Samples were then centrifuged at max speed and the supernatant analyzed on the analytical HPLC column (HPLC method). Compounds $\mathbf{3 a}$ and $\mathbf{3 b}$ had a retention time of 15.25 and $14.65 \mathrm{~min}$, respectively. Monitoring was at $750 \mathrm{~nm}$ and the area of any observed peak was assessed in each HPLC chromatogram. Bar plot representation of the compound stability is shown as \%intact (Eq. (1)), where

$$
\% \text { intact }=\frac{\text { peak area of compound } 3}{\sum \text { Areas of all observed peaks }} \times 100 .
$$

In vitro fluorescence and photoacoustic characterization. Mouse whole blood was collected through cardiac puncture and stored in $1.5 \mathrm{~mL}$ heparin-lithiumcoated tubes (Eppendorf, cat no.: 022379208) on ice and used within $2 \mathrm{~h}$ of collection. A serial dilution of Cy7-1-maltotriose was prepared in either PBS (fluorescence and PAI) or murine whole blood (PAI). In the IVIS spectrum instrument, fluorescence images of 50, 25, 10, 1, 0.5, $0 \mu \mathrm{M}$ solution of Cy7-1-maltotriose in PBS were collected in a Corning ${ }^{\circ}$ black clear bottom 96 -well plate. While in PAI, the samples were measured in a Vevo PHANTOM imaging chamber, where the samples were injected inside the tubes and imaging conducted using the Vevo3100 LAZR imaging system. Similarly, photoacoustic measurements of 100, 50, 25, 10, 1, and $0 \mu \mathrm{M}$ solution of Cy7-1-maltotriose in whole blood were collected after the tubes were flushed with heparin sodium solution (1000 USP/mL, NDC 63739-93114) to avoid coagulation of blood inside the tube.

Bacterial cultures. E. coli was obtained from American Type Culture Collections (ATCC 33456). E. coli mutants JW3992-1 (LamB and MalG deficient), JW3995 (LamB and Mal K deficient), and JW1613 (LamB and Mal X-PTS permease deficient) were obtained from E. coli Genetic Resources at Yale (Yale University, New Haven, USA). Bioluminescent strain of Pseudomonas aeruginosa (Xen 5), Bacillus subtilis, and Staphylococcus aureus (Xen 36) were obtained from Perkin Elmer.

Overnight culture conditions. E. coli overnight $(\mathrm{O} / \mathrm{N})$ culture was prepared by inoculating a colony in Luria-Bertani (LB) broth $(3 \mathrm{~mL})$ in an incubator shaker at $37^{\circ} \mathrm{C}$. The mutant E. coli strains and Xen 36, the bioluminescent strain of S. aureus, were grown in LB with $50 \mu \mathrm{g} / \mathrm{mL}$ of Kanamycin. After $16 \mathrm{~h}, 600 \mu \mathrm{L}$ of the O/N culture was added to $30 \mathrm{~mL}$ of $\mathrm{LB}$ in a $200 \mathrm{~mL}$ flask and placed in an incubator shaker at $37^{\circ} \mathrm{C}$ until the bacterial culture reached the log phase $\left(\mathrm{OD}_{600}=0.5\right)$. Metabolically inactive E. coli was prepared by either treating $\mathrm{O} / \mathrm{N}$ culture $\left(\mathrm{OD}_{600}=\right.$ $0.5)$ with sodium azide $(10 \mathrm{mM})$ and incubating for $1 \mathrm{~h}$ in an incubator shaker at $37^{\circ} \mathrm{C}$ or by heating the culture to $90^{\circ} \mathrm{C}$ for $30 \mathrm{~min}$. All cultures were harvested by centrifugation and pellets washed three times with HBSS $(1 \times)$ before suspending at the concentration of interest.

Bacteria competition assay. Aliquots of $10^{8} \mathrm{CFU}$ of E. coli were first incubated with test compounds $(1 \mathrm{mM})$ for $1 \mathrm{~h}$ at $37^{\circ} \mathrm{C}$. After the first incubation, the bacteria culture was centrifuged at $9400 \times g$ for $5 \mathrm{~min}$, and the pellet washed with HBSS (1 $\times$ ) three times. Pellets were then resuspended in a solution of ${ }^{3} \mathrm{H}$-maltose $(1 \mu \mathrm{Ci}$ in $200 \mu \mathrm{L}$ HBSS (1×); American Radiolabeled Chemicals, Inc., St. Louis, MO, USA) and incubated for $30 \mathrm{~min}$ at $37^{\circ} \mathrm{C}$. Aliquots were then centrifuged and washed with HBSS $(1 \times)$ three times before lysing in a bacterial lysis buffer (BugBuster, EMD, Billerica MA USA). Activity in bacteria lysates was then counted in a gamma-counter and protein concentration determined using a bicinchoninic acid (BCA) assay (Pierce, Thermo Fisher Scientific). Test compounds included maltose as a positive control, azide-1-maltotriose and Cy7-1-maltotriose. In addition, aliquots incubated with only ${ }^{3} \mathrm{H}$-maltose were also included to assess normal uptake. Results are shown as counts per minute (CPM) normalized to protein content ( $\mu$ g of protein) per sample ( $n=3$ per study).

Bacteria uptake studies. Aliquots of $10^{8} \mathrm{CFU}$ of E. coli, E. coli mutants, azideinactivated E. coli, Pseudomonas aeruginosa, Bacillus subtilis, and Staphylococcus aureus (Xen 36) were incubated with the same amount of compound $\mathbf{3 a}$ or $\mathbf{3 b}$ for $1 \mathrm{~h}$ at $37^{\circ} \mathrm{C}$. Aliquots were then centrifuged $(9400 \times g$ for $5 \mathrm{~min})$ and washed with HBSS (1 $\times$ ) three times before lysing in a bacteria lysis buffer (BugBuster for E. coli and mutants, EMD; B-PER ${ }^{\text {mi }}$ Complete Bacterial Protein Extraction Reagent for the rest of strains, Thermo Scientific $\left.{ }^{\mathrm{w} x}\right)$. The fluorescence intensity in bacteria lysates was measured in a SpectraMax GEMINI EM fluorescent plate reader (Molecular Devices, San Jose, CA, USA).

In vitro influx studies. Aliquots of $10^{8} \mathrm{CFU}$ of E. coli were incubated with the same amount of compound $\mathbf{3 a}$ or $3 \mathbf{b}(50 \mu \mathrm{M})$ at $37^{\circ} \mathrm{C}$. After each time point $(30$, $60,240$, and $1080 \mathrm{~min})$, aliquots were then centrifuged $(9400 \times g$ for $5 \mathrm{~min})$ and washed with HBSS $(1 \times)$ three times before lysing in a bacteria lysis buffer (BugBuster for E. coli and mutants, EMD; B-PER ${ }^{\mathrm{m}}$ Complete Bacterial Protein Extraction Reagent for the rest of strains, Thermo Scientific $\left.{ }^{\mathrm{m}}\right)$. The fluorescence intensity in bacteria lysates was measured in a SpectraMax GEMINI EM fluorescent plate reader (Molecular Devices, San Jose, CA, USA).

Animals and infection models. All animal models and in vivo experiments were approved by the Stanford University Institutional Animal Care and Use Committee.

E. coli-induced myositis murine model. Female $n u / n u$ mice 6-7 weeks old were anesthetized by isoflurane inhalation (2-3\%). $10^{8} \mathrm{CFU}$ of E. coli in $50 \mu \mathrm{L}$ of HBSS was injected intramuscularly into the right thigh muscle of the mice. As a control, $10^{8} \mathrm{CFU}$ of heat-inactivated E. coli or E. coli MalG+LamB mutant in $50 \mu \mathrm{L}$ of HBSS $(1 \times)$ was injected intramuscularly in the left thigh.

Staphylococcus aureus wound infection murine model. Female CD1 or SKH1elite mice, 6-8 weeks old were anesthetized by isoflurane inhalation (2-3\%). A small wound on the upper back or lower back for the CD1 or SKH1 mice, respectively, was formed using a sharp pair of scissors. Staphylococcus aureus in $20 \mu \mathrm{L}$ saline was inoculated into a small pocket subcutaneously before sealing the wound with Vetbond adhesive (1469SB; 3M, St. Paul, MN, USA).

Fluorescence imaging in $\boldsymbol{E}$. coli-induced myositis model. Immediately after E. coli-induced murine myositis $(n=3)$, Cy7-1-maltotriose ( $5 \mathrm{nmol}$ in $2 \% \mathrm{DMSO} /$ saline, $100 \mu \mathrm{L}$ ) was injected via the tail vein. Fluorescence images were captured at 
$1,3,5$, and $20 \mathrm{~h}$ post-injection of Cy7-1-maltotriose. The fluorescence intensity in the right thigh muscle (E. coli) and left thigh muscle (heat-inactivated E. coli) were then quantified and analyzed.

PAI in E. coli-induced myositis model. Upon inducing myositis by intramuscular injection of $10^{8} \mathrm{CFU}$ of $E$. coli in the right thigh muscle of nu/nu mice, mice were anesthetized and fixed in the prone position for PAI. PA and US images were then collected using a Vevo2100 LAZR imaging system (Vevo ${ }^{\circ}$ LAZR, VisualSonics, Inc., Canada) with PA-mode and Ultrasound B-mode, respectively. The PA system was connected to an LZ-250 transducer that delivered the nanosecond laser pulses with a wavelength of $700 \mathrm{~nm}$. A cross-sectional two-dimensional (2D) and 3D images of the right and left thigh muscle were acquired, and 3D rendered images presented as colored photoacoustic layer overlaid on top of the ultrasound images were produced using VevoLAB software (VisualSonics, Inc., Canada). Using MATLAB, the total PA signal intensity of the $3 \mathrm{D}$ volume was produced and quantified using Fiji software and presented as a.u. PA and US images were collected before $(n=5)$ and $24 \mathrm{~h}$ after $(n=4)$ tail vein injection of Cy7-1-maltotriose ( $5 \mathrm{nmol}$ in $2 \% \mathrm{DMSO} /$ saline, $100 \mu \mathrm{L}$ injection)

Fluorescence imaging comparison between compounds $\mathbf{3 a}$ and $\mathbf{3 b}$. Immediately after E. coli-induced murine myositis, Cy7-1-maltotriose (compound 3a, $n=$ 6) or Cy7-1-maltohexose (compound $\mathbf{3 b}, n=4)(5 \mathrm{nmol}$ in $2 \% \mathrm{DMSO} / \mathrm{saline}$, $100 \mu \mathrm{L}$ injection) were injected via the tail vein. Fluorescence images were captured at 2, 4, and $18 \mathrm{~h}$ post-injection of the probes. The fluorescence intensity in the right thigh muscle (E. coli) and left thigh muscle (control) were quantified and data analyzed. The data are presented as the ratio of fluorescence intensity in the right thigh muscle (infected) vs left thigh muscle (control).

PAI comparison between compounds $\mathbf{3 a}$ and $\mathbf{3 b}$. After FLI, PA, and US images were collected post-injection of either $\mathbf{3} \mathbf{a}(n=6)$ or $\mathbf{3} \mathbf{b}(n=3)$ using a Vevo2 100 LAZR imaging system (Vevo LAZR, VisualSonics, Inc., Canada) with PA-mode and Ultrasound B-mode, respectively. The PA system was connected to an LZ250 transducer that delivered the nanosecond laser pulses with a wavelength of $700 \mathrm{~nm}$. A cross-sectional 2D and 3D images of the right and left thigh muscle were acquired, and $3 \mathrm{D}$ rendered images presented as colored photoacoustic layer overlaid on top of the ultrasound images were produced using VevoLAB software (VisualSonics, Inc., Canada). Using MATLAB, the total PA signal intensity of the 3D volume was produced and quantified using Fiji software and presented as a.u.

In vivo specificity study to maltodextrin transporter. Myositis in mouse was induced by injecting $10^{8} \mathrm{CFU}$ of $E$. coli and $10^{8} \mathrm{CFU}$ of E. coli MalG+LamB mutant in the right and left thigh, respectively $(n=5)$. Cy7-1-maltotriose $(10 \mathrm{nmol}$ in $2 \% \mathrm{DMSO} /$ Saline, $200 \mu \mathrm{L}$ injection) was then injected via the tail vein. Fluorescence images were acquired 3 and $20 \mathrm{~h}$ post-injection. After imaging, mice were sacrificed, both right and left thigh muscle collected, and ex vivo fluorescence images acquired.

In vitro imaging of $\boldsymbol{S}$. aureus-infected biomaterial. Sterile catheters were collected from a BD Insyte ${ }^{\mathrm{m}}$ Autoguard ${ }^{\mathrm{m} w}$ BC Shielded IV Catheter (ref: 382544, BD, Franklin Lakes, NJ, USA) and placed in a $10^{6} \mathrm{CFU}$ of S. aureus per $\mathrm{mL}$ solution in an incubator shaker at $37^{\circ} \mathrm{C}$ for $2 \mathrm{~h}$. Catheters were then placed in a Cy7-1maltotriose solution $(50 \mathrm{nmol} / \mathrm{mL})$ and incubated for $37^{\circ} \mathrm{C}$ for $1 \mathrm{~h}$ before washing by gently dipping in PBS (1×) solution. As a control, sterile catheters that were not exposed to infection or infected catheters that were not incubated with the probe were assessed. BLI and fluorescence images of the catheters were then collected and analyzed. In addition, the catheters were placed inside a $4 \%$ agarose phantom and axial ultrasound and photoacoustic images collected using a Vevo2100 LAZR imaging system (Vevo ${ }^{\circledR}$ LAZR, VisualSonics, Inc., Toronto, Canada) and irradiation at $700 \mathrm{~nm}$. PA and US images were analyzed using VevoLAB software (VisualSonics, Inc., Toronto, Canada).

Fluorescence and bioluminescence imaging of treatment study. $10^{6} \mathrm{CFU}(n=$ 9) of kanamycin-resistant $S$. aureus were inoculated subcutaneously in the wound in SKH1-elite mice. Mice were then administered kanamycin $(800 \mathrm{mg} / \mathrm{kg})$ intramuscularly once daily to ensure no other bacterial infections occur. Two days postsurgery, Cy7-1-maltotriose ( $10 \mathrm{nmol}$ in $2 \% \mathrm{DMSO} /$ saline, $200 \mu \mathrm{L}$ injection) was injected via the tail vein and bioluminescence and fluorescence imaging were performed at $19,45,69,93$, and $144 \mathrm{~h}$ post-injection. Following imaging at $19 \mathrm{~h}$, the mice were divided into untreated $(n=4)$ and treated $(n=5)$ groups where the first group only received kanamycin and the latter was administered vancomycin $(110 \mathrm{mg} / \mathrm{kg})$ subcutaneously twice daily in addition to kanamycin. After 7 days of antibiotic treatment, mice were injected again with Cy7-1-maltotriose $(10 \mathrm{nmol}$ in $2 \% \mathrm{DMSO} / \mathrm{saline}, 200 \mu \mathrm{L}$ injection) and bioluminescence and fluorescence imaging performed $24 \mathrm{~h}$ post-injection.

PAI of treatment study. Twenty and $25 \mathrm{~h}$ post-injection of Cy7-1-maltotriose (before and after treatment respectively), mice were anesthetized and fixed in the prone position for PAI. PA and US images were then collected using a Vevo3100 LAZR imaging system (Vevo ${ }^{\circ}$ LAZR, VisualSonics, Inc., Canada) with PA-mode and B-mode, respectively. The PA system connected to an MX-250 transducer that delivered the nanosecond laser pulses with a wavelength of $700 \mathrm{~nm}$. A crosssectional 2D and 3D images of the right and left thigh muscle were acquired, and $3 \mathrm{D}$ rendered images presented as colored photoacoustic layer overlaid on top of the ultrasound images were produced using VevoLAB software (VisualSonics, Inc., Canada). The whole region of the wound was highlighted using VevoLAB software and average PA signal intensity quantified and presented as a.u.

Bacterial burden differentiation. $10^{8} \mathrm{CFU}(n=5), 10^{6} \mathrm{CFU}(n=3)$, and $10^{4} \mathrm{CFU}$ $(n=3)$ of $S$. aureus were inoculated subcutaneously in the wound in CD1 mice. Cy7-1-maltotriose ( $5 \mathrm{nmol}$ in $2 \% \mathrm{DMSO} / \mathrm{saline}, 100 \mu \mathrm{L}$ injection) was then injected via the tail vein. To ensure no other infections occur, a daily dose of kanamycin $(800 \mathrm{mg} / \mathrm{kg})$ was given to the mice intramuscularly. Fluorescence and bioluminescence images were then captured at 5 and $20 \mathrm{~h}$ post-injection of the probe. Image analysis was conducted using Living Image software.

Reporting summary. Further information on research design is available in the Nature Research Reporting Summary linked to this article.

\section{Data availability}

Data supporting the findings of this work are available within the paper and its Supplementary Information files. A reporting summary for this article is available as a Supplementary Information file. The datasets generated and analyzed in the current study are available from the corresponding author on request. The source data underlying Figs. $2 \mathrm{a}-\mathrm{c}, 3 \mathrm{~b}, \mathrm{~d}, 4 \mathrm{~b}, \mathrm{~d}, 5 \mathrm{~b}, \mathrm{~d}, 6 \mathrm{c}, \mathrm{d}$, and $7 \mathrm{~b}$ are provided as a Source Data file.

\section{Code availability}

The codes that support the findings of this study are available from https://github.com/ idanstei/Maltotriose-based-probes-for-fluorescence-and-photoacoustic-imaging-ofbacterial-infections.

Received: 12 November 2018; Accepted: 13 February 2020; Published online: 06 March 2020

\section{References}

1. Fiore, D. C., Fettic, L. P., Wright, S. D. \& Ferrara, B. R. Antibiotic overprescribing: still a major concern. J. Fam. Pract. 66, 730-736 (2017)

2. McKenna, M. Antibiotic resistance: the last resort. Nature 499, 394-396 (2013).

3. Sporer, S. M., Weinstein, J. N. \& Koval, K. J. The geographic incidence and treatment variation of common fractures of elderly patients. J. Am. Acad. Orthop. Surg. 14, 246-255 (2006).

4. Edwards, R. \& Harding, K. G. Bacteria and wound healing. Curr. Opin. Infect Dis. 17, 91-96 (2004).

5. Bode, L. G. M. et al. Preventing surgical-site infections in nasal carriers of Staphylococcus aureus. N. Engl. J. Med. 362, 9-17 (2010).

6. Arciola, C. R., Campoccia, D. \& Montanaro, L. Implant infections: adhesion biofilm formation and immune evasion. Nat. Rev. Microbiol. 16, 397-409 (2018).

7. Anderson, D. J. Surgical site infections. Infect. Dis. Clin. North Am. 25, 135-153 (2011).

8. Anderson, D. J. \& Kaye, K. S. Staphylococcal surgical site infections. Infect. Dis. Clin. North Am. 23, 53-72 (2009).

9. Trampuz, A. \& Zimmerli, W. Diagnosis and treatment of infections associated with fracture-fixation devices. Injury 37, S59-S66 (2006).

10. Jain, S. K. The promise of molecular imaging in the study and treatment of infectious diseases. Mol. Imaging Biol. 19, 341-347 (2017).

11. Sevick-Muraca, E. M. Translation of near-infrared fluorescence imaging technologies: emerging clinical applications. Annu. Rev. Med. 63, 217-231 (2012).

12. James, M. L. \& Gambhir, S. S. A molecular imaging primer: modalities, imaging agents, and applications. Physiol. Rev. 92, 897-965 (2012)

13. Wang, L. V. \& Hu, S. Photoacoustic tomography: in vivo imaging from organelles to organs. Science 335, 1458-1462 (2012).

14. Mallidi, S., Luke, G. P. \& Emelianov, S. Photoacoustic imaging in cance detection, diagnosis, and treatment guidance. Trends Biotechnol. 29, 213-221 (2011).

15. Beard, P. Biomedical photoacoustic imaging. Interface Focus 1, 602-631 (2011).

16. Steinberg, I. et al. Photoacoustic clinical imaging. Photoacoustics 14, 77-98 (2019). 
17. Zhang, E. Z., Laufer, J. G., Pedley, R. B. \& Beard, P. C. In vivo high-resolution 3D photoacoustic imaging of superficial vascular anatomy. Phys. Med. Biol. 54, 1035-1046 (2009).

18. Mari, J. M., Xia, W., West, S. J. \& Desjardins, A. E. Interventional multispectral photoacoustic imaging with a clinical ultrasound probe for discriminating nerves and tendons: an ex vivo pilot study. J. Biomed. Opt. 20, 110503 (2015).

19. van Oosten, M. et al. Real-time in vivo imaging of invasive- and biomaterialassociated bacterial infections using fluorescently labelled vancomycin. Nat. Commun. 4, 2584 (2013).

20. Li, L. L. et al. Pathological-condition-driven construction of supramolecular nanoassemblies for bacterial infection detection. Adv. Mater. 28, 254-262 (2016).

21. Wang, Y. et al. Preclinical evaluation of photoacoustic imaging as a novel noninvasive approach to detect an orthopaedic implant infection. J. Am. Acad. Orthop. Surg. 25, S7-S12 (2017).

22. Tang, E. N., Nair, A., Baker, D. W., Hu, W. \& Zhou, J. In vivo imaging of infection using a bacteria-targeting optical nanoprobe. J. Biomed. Nanotechnol. 10, 856-863 (2014).

23. Romero Pastrana, F. et al. Noninvasive optical and nuclear imaging of Staphylococcus-specific infection with a human monoclonal antibody-based probe. Virulence 9, 262-272 (2018).

24. Kwon, H.-Y. et al. Development of a universal fluorescent probe for grampositive bacteria. Angew. Chem. Int. Ed. Engl. 58, 8426-8431 (2019).

25. Zhou, J., Qi, G.-B. \& Wang, H. A purpurin-peptide derivative for selective killing of gram-positive bacteria via insertion into cell membrane. J. Mater. Chem. B 4, 4855-4861 (2016).

26. Yao, J. et al. Multiscale photoacoustic tomography using reversibly switchable bacterial phytochrome as a near-infrared photochromic probe. Nat. Methods 13, 67-73 (2015).

27. Chee, R. K. W., Li, Y., Zhang, W., Campbell, R. E. \& Zemp, R. J. In vivo photoacoustic difference-spectra imaging of bacteria using photoswitchable chromoproteins. J. Biomed. Opt. 23, 106006 (2018).

28. Jiang, Y. et al. Violacein as a genetically-controlled, enzymatically amplified and photobleaching-resistant chromophore for optoacoustic bacterial imaging. Sci. Rep. 5, 11048 (2015).

29. Peters, L. et al. Phototrophic purple bacteria as optoacoustic in vivo reporters of macrophage activity. Nat. Commun. 10, 1191 (2019).

30. Ning, X. et al. Maltodextrin-based imaging probes detect bacteria in vivo with high sensitivity and specificity. Nat. Mater. 10, 602-607 (2011).

31. Ning, X. et al. PET imaging of bacterial infections with fluorine-18-labeled maltohexaose. Angew. Chem. Int. Ed. Engl. 53, 14096-14101 (2014).

32. Takemiya, K. et al. Novel PET and near infrared imaging probes for the specific detection of bacterial infections associated with cardiac devices. JACC Cardiovasc. Imaging 12, 875-886 (2019).

33. Pang, X. et al. Bacteria-responsive nanoliposomes as smart sonotheranostics for multidrug resistant bacterial infections. ACS Nano. https://doi.org/ 10.1021/acsnano.8b09336 (2019).

34. Gowrishankar, G. et al. Investigation of 6-[ $\left.{ }^{1} 8 \mathrm{~F}\right]$-fluoromaltose as a novel PET tracer for imaging bacterial infection. PLOS ONE 9, e107951 (2014).

35. Namavari, M., Gowrishankar, G., Hoehne, A., Jouannot, E. \& Gambhir, S. S. Synthesis of $\left[{ }^{18} \mathrm{~F}\right]$-labelled maltose derivatives as PET tracers for imaging bacterial infection. Mol. Imaging Biol. 17, 168-176 (2015).

36. Gowrishankar, G. et al. Specific imaging of bacterial infection using $6^{\prime \prime}-18 \mathrm{~F}$ fluoromaltotriose: a second generation PET tracer targeting the maltodextrin transporter in bacteria. J. Nucl. Med. 58, 1679-1684 (2017).

37. Khamsi, J., Ashmus, R. A., Schocker, N. S. \& Michael, K. A high-yielding synthesis of allyl glycosides from peracetylated glycosyl donors. Carbohydr. Res. 357, 147-150 (2012).

38. Axer, A. et al. Harnessing the maltodextrin transport mechanism for targeted bacterial imaging: structural requirements for improved in vivo stability in tracer design. ChemMedChem 13, 241-250 (2018).

39. Deán-Ben, X. L. \& Razansky, D. Portable spherical array probe for volumetric real-time optoacoustic imaging at centimeter-scale depths. Opt. Express 21, 28062-28071 (2013).

40. Daoudi, K. et al. Handheld probe integrating laser diode and ultrasound transducer array for ultrasound/photoacoustic dual modality imaging. Opt. Express 22, 26365-26374 (2014).

41. Kim, J. et al. Programmable real-time clinical photoacoustic and ultrasound imaging system. Sci. Rep. 6, 35137 (2016).

42. Dippel, R. \& Boos, W. The maltodextrin system of Escherichia coli: metabolism and transport. J. Bacteriol. 187, 8322-8331 (2005).

43. Dutzler, R., Wang, Y. F., Rizkallah, P., Rosenbusch, J. P. \& Schirmer, T. Crystal structures of various maltooligosaccharides bound to maltoporin reveal a specific sugar translocation pathway. Structure 4, 127-134 (1996).

44. Quiocho, F. A., Spurlino, J. C. \& Rodseth, L. E. Extensive features of tight oligosaccharide binding revealed in high-resolution structures of the maltodextrin transport/chemosensory receptor. Structure 5, 997-1015 (1997).
45. Oldham, M. L., Chen, S. \& Chen, J. Structural basis for substrate specificity in the Escherichia coli maltose transport system. Proc. Natl Acad. Sci. USA 110, 18132-18137 (2013)

46. Sauvageot, N. et al. Enterococcus faecalis uses a PTS permease and a host colonization-related $\mathrm{ABC}$ transporter for maltodextrin uptake. J. Bacteriol. 199, e00878 (2017).

47. Licht, A. et al. Structural and functional characterization of a maltose/ maltodextrin $\mathrm{ABC}$ transporter comprising a single solute binding domain (MalE) fused to the transmembrane subunit MalF. Res. Microbiol. 170, 1-12 (2019).

48. Dumont, E. et al. Mechanistic aspects of maltotriose-conjugate translocation to the gram-negative bacteria cytoplasm. Life Sci. Alliance 2, e201800242 (2019).

49. Kurtz, S. M., Ong, K. L., Lau, E. \& Bozic, K. J. Impact of the economic downturn on total joint replacement demand in the United States: updated projections to 2021. J. Bone Jt. Surg. Am. 96, 624-630 (2014).

50. Pitta, M. et al. Failure after modern total knee arthroplasty: a prospective study of 18,065 knees. J. Arthroplast. 33, 407-414 (2018).

51. Fernández-Sampedro, M. et al. Accuracy of different diagnostic tests for early, delayed and late prosthetic joint infection. BMC Infect. Dis. 17, 592 (2017).

52. Arciola, C. R., Montanaro, L. \& Costerton, J. W. New trends in diagnosis and control strategies for implant infections. Int J. Artif. Organs 34, 727-736 (2011).

53. Harris, D. L. G. et al. Rapid identification of Staphylococci from prosthetic joint infections using MALDI-TOF mass-spectrometry. Int J. Artif. Organs 33, 568-574 (2010).

54. Deurenberg, R. H. et al. Application of next generation sequencing in clinical microbiology and infection prevention. J. Biotechnol. 243, 16-24 (2017).

55. Tagini, F. \& Greub, G. Bacterial genome sequencing in clinical microbiology: a pathogen-oriented review. Eur. J. Clin. Microbiol. Infect. Dis. 36, 2007-2020 (2017).

56. van Belkum, A., Welker, M., Pincus, D., Charrier, J. P. \& Girard, V. Matrixassisted laser desorption ionization time-of-flight mass spectrometry in clinical microbiology: what are the current issues? Ann. Lab. Med. 37, 475-483 (2017).

57. Li, L. et al. Single-impulse panoramic photoacoustic computed tomography of small-animal whole-body dynamics at high spatiotemporal resolution. Nat. Biomed. Engl. 1, 977-11 (2017)

58. Merčep, E., Herraiz, J. L., Deán-Ben, X. L. \& Razansky, D. Transmission-reflection optoacoustic ultrasound (TROPUS) computed tomography of small animals. Light Sci. Appl. 8, 1-12 (2019).

59. Kim, C., Erpelding, T. N., Jankovic, L. \& Wang, L. V. Performance benchmarks of an array-based hand-held photoacoustic probe adapted from a clinical ultrasound system for non-invasive sentinel lymph node imaging. Philos. Trans. R. Soc. A 369, 4644-4650 (2011).

60. Sivasubramanian, K. \& Pramanik, M. High frame rate photoacoustic imaging at 7000 frames per second using clinical ultrasound system. Biomed. Opt. Express 7, 312-323 (2016)

61. Steinberg, I., Shiloh, L., Gannot, I. \& Eyal, A. First-in-human study of bone pathologies using low-cost and compact dual-wavelength photoacoustic system. IEEE J. Sel. Top. Quant. Electron. 25, https://doi.org/10.1109/ JSTQE.2018.2866702 (2018)

\section{Acknowledgements}

Dr. Derek Holman for providing training on the mouse wound model. Dr. Mohammad Namavari for providing azide-6"-maltotriose intermediate. Dr. Hui-Yen Chuang for assisting with bacterial culture. Dr. Jonathan Hardy and Dr. Christopher H. Contag for providing the Xen 36 strains. The Stanford University Small Animal Imaging Facility for training and help with small-animal imaging studies. Dr. Naoki Nishio, Dr. Nynke S. van den Berg, Dr. Katheryne Wilson, and Dr. Eben Rosenthal for assistance and allowing us to use their Vevo3100 LAZR system.

\section{Author contributions}

A.Z. conducted all the synthesis and characterization of the probes, designed, carried out and analyzed experiments, and wrote the manuscript. G.G. designed and carried out experiments and contributed to the writing of the manuscript. I.S. wrote the code for PA imaging analysis and assisted with PA image analysis, production, scientific discussion and manuscript editing. T.H. assisted with compound stability and metabolism studies and manuscript editing. S.S.G. designed and supervised the project and contributed to the writing of the manuscript.

\section{Competing interests}

S.S.G. declares competing financial interests with Visualsonics Inc. The rest of the authors declare no competing interests. 


\section{Additional information}

Supplementary information is available for this paper at https://doi.org/10.1038/s41467020-14985-8

Correspondence and requests for materials should be addressed to S.S.G.

Peer review information Nature Communications thanks the anonymous reviewers for their contribution to the peer review of this work. Peer reviewer reports are available.

Reprints and permission information is available at http://www.nature.com/reprints

Publisher's note Springer Nature remains neutral with regard to jurisdictional claims in published maps and institutional affiliations. (c) (i) Open Access This article is licensed under a Creative Commons Attribution 4.0 International License, which permits use, sharing, adaptation, distribution and reproduction in any medium or format, as long as you give appropriate credit to the original author(s) and the source, provide a link to the Creative Commons license, and indicate if changes were made. The images or other third party material in this article are included in the article's Creative Commons license, unless indicated otherwise in a credit line to the material. If material is not included in the article's Creative Commons license and your intended use is not permitted by statutory regulation or exceeds the permitted use, you will need to obtain permission directly from the copyright holder. To view a copy of this license, visit http://creativecommons.org/ licenses/by/4.0/.

(C) The Author(s) 2020 